\title{
Two-photon transitions in hydrogen and cosmological recombination ${ }^{\star}$
}

\author{
J. Chluba ${ }^{1}$ and R. A. Sunyaev ${ }^{1,2}$ \\ 1 Max-Planck-Institut für Astrophysik, Karl-Schwarzschild-Str. 1, 85741 Garching bei München, Germany \\ e-mail: jchluba@mpa-garching.mpg.de \\ 2 Space Research Institute, Russian Academy of Sciences, Profsoyuznaya 84/32, 117997 Moscow, Russia
}

Received 21 May 2007 / Accepted 22 November 2007

\begin{abstract}
We study the two-photon process for the transitions $n \mathrm{~s} \rightarrow 1 \mathrm{~s}$ and $n \mathrm{~d} \rightarrow 1 \mathrm{~s}$ in hydrogen up to large $n$. For $n \leq 20$ we provide simple analytic fitting formulae to describe the non-resonant part of the two-photon emission profiles. Combining these with the analytic form of the cascade-term yields a simple and accurate description of the full two-photon decay spectrum, which only involves a sum over a few intermediate states. We demonstrate that the cascade term naturally leads to a nearly Lorentzian shape of the two-photon profiles in the vicinity of the resonances. However, due to quantum-electrodynamical corrections, the two-photon emission spectra deviate significantly from the Lorentzian shape in the very distant wings of the resonances. We investigate up to which distance the two-photon profiles are close to a Lorentzian and discuss the role of the interference term. We then analyze how the deviation of the two-photon profiles from the Lorentzian shape affects the dynamics of cosmological hydrogen recombination. Since in this context the escape of photons from the Lyman- $\alpha$ resonance plays a crucial role, we concentrate on the two-photon corrections in the vicinity of the Lyman- $\alpha$ line. Our computations show that the changes in the ionization history due to the additional two-photon process from high shell $(n>2)$ likely do not reach the percent-level. For conservative assumptions we find a correction $\Delta N_{\mathrm{e}} / N_{\mathrm{e}} \sim-0.4 \%$ at redshift $z \sim 1160$. This is numerically similar to the result of another recent study; however, the physics leading to this conclusion is rather different. In particular, our calculations of the effective two-photon decay rates yield significantly different values, where the destructive interference of the resonant and non-resonant terms plays a crucial role in this context. We also show that the bulk of the corrections to the ionization history is only due to the $3 \mathrm{~s}$ and $3 \mathrm{~d}$-states and that the higher states do not contribute significantly.
\end{abstract}

Key words. atomic processes - atomic data - radiation mechanisms: general - cosmology: theory - cosmic microwave background

\section{Introduction}

During the epoch of cosmological hydrogen recombination (typical redshifts $800 \lesssim z \lesssim 1600$ ), any direct recombination of electrons to the ground state of hydrogen is immediately followed by the ionization of a neighboring neutral atom due to re-absorption of the newly released Lyman-continuum photon. In addition, because of the enormous difference in the $2 p \leftrightarrow 1$ s dipole transition rate and the Hubble expansion rate, photons emitted close to the center of the Lyman- $\alpha$ line scatter $\sim 10^{8}-10^{9}$ times before they can finally escape further interaction with the medium and thereby permit a successful settling of electrons in the hydrogen ground state. It is due to these very peculiar circumstances that the $2 \mathrm{~s} \leftrightarrow 1 \mathrm{~s}$-two-photon decay process, which is $\sim 10^{8}$ orders of magnitude slower than the Lyman- $\alpha$ resonance transition, is able to substantially control the dynamics of cosmological hydrogen recombination (Zeldovich et al. 1968; Peebles 1968), allowing about $57 \%$ of all hydrogen atoms in the Universe to recombine at redshift $z \lesssim 1400$ (Chluba \& Sunyaev 2006a).

The tremendous success in observations of the cosmic microwave background temperature and polarization anisotropies (Hinshaw et al. 2006; Page et al. 2006) has recently motived several works on high precision computations of the cosmological hydrogen (Dubrovich \& Grachev 2005; Chluba \& Sunyaev 2006b; Kholupenko \& Ivanchik 2006; Novosyadlyj 2006;

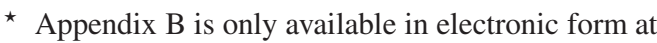
http://www . aanda.org
}

Rubiño-Martín et al. 2006; Chluba et al. 2007; Wong \& Scott 2007; Chluba \& Sunyaev 2007b) and helium (Switzer \& Hirata 2007a; Hirata \& Switzer 2007; Switzer \& Hirata 2007b; Kholupenko et al. 2007) recombination history.

One interesting additional physical process, which had been neglected in earlier computations (Seager et al. 1999, 2000), is connected to the two-photon transitions from high $n \mathrm{~s}$ and $n d$-states to the ground state of hydrogen and was first proposed by Dubrovich \& Grachev (2005). In their computations a simple scaling for the total two-photon decay rate of the $\mathrm{s}$ and d-states in hydrogen was given and, including these additional channels leading to the $1 \mathrm{~s}-$ level, corrections to the ionization history were found that exceed the percent-level. These modifications would have a strong impact on the determination of the key cosmological parameters (Lewis et al. 2006) and therefore require careful consideration.

More recently, theoretical values for the non-resonant ${ }^{1}$ twophoton decay rates of the $3 \mathrm{~s}$ and $3 \mathrm{~d}$ level based on the work of Cresser et al. (1986) were utilized to improve the computations of Dubrovich \& Grachev (2005), showing that the effect of two-photon transitions from higher levels on the recombination history is likely to be less than $\sim 0.4 \%$ (Wong \& Scott 2007). However, to our knowledge, neither extensive calculations of the two-photon decay rates for the transitions $n \mathrm{~s} \rightarrow 1 \mathrm{~s}$ and $n \mathrm{~d} \rightarrow 1 \mathrm{~s}$ from high levels exist nor detailed reports of direct

\footnotetext{
1 We base our definition of this terminus on the energies of the involved intermediate states (see Sect. 2 for details).
} 
measurements can be found in the literature, so that Wong \& Scott (2007) also had to extrapolate existing values towards higher levels, largely relying on the previous estimates by Dubrovich \& Grachev (2005) and the values given in Cresser et al. (1986). Here we argue that, due to quantum-interference, it is difficult to separate the contributions of the pure two-photon process from the resonant single photon processes. Therefore to answer the question how much two-photon processes are affecting the recombination history requires a more rigorous treatment in connection with the radiative transfer and escape of photons (Varshalovich \& Syunyaev 1968; Grachev \& Dubrovich 1991; Rybicki \& dell'Antonio 1994; Chluba \& Sunyaev 2007a) from the main resonances (especially the Lyman- $\alpha$ line). Also Hirata $\&$ Switzer (2007) re-analyzed the importance of the two-photon process in the context of cosmological helium recombination and showed that, for high values of $n$, the rate estimates by Dubrovich \& Grachev (2005) are rather rough and that in particular the linear scaling with $n$ fails. Here we provide some conservative lower limits on the possible impact of the two-photon transitions on the hydrogen recombination history. We show that the non-resonant contribution to the two-photon decay rate indeed scales $\propto n$ (see Sect. 4.3.1). However, due to destructive interference between the resonant and non-resonant terms, the effective two-photon decay rate is much lower and actually decreases with $n$.

If one considers an isolated neutral hydrogen atom ${ }^{2}$ with the electron in some excited state $(n, l)$, then because of the finite lifetime of the level, the electron will reach the ground-state after some short time (typically $\sim 10^{-8} \mathrm{~s}$ ), in general releasing more than one photon. Astrophysicists usually describe this multiphoton cascade, also known as Seaton-cascade, as a sequence of independent, single-step, one-photon processes (e.g. see Seaton 1959), where every resonance has a pure Lorentzian shape. This approximation should be especially good in the presence of many perturbing particles (free electrons and ions), such as in stellar atmospheres, which destroy the coherence of processes ${ }^{3}$ involving more than one intermediate transition. However, in extremely low density environments, like the expanding Universe during cosmological hydrogen recombination, hardly any perturbing particle is within the Weisskopf-radius (Weisskopf 1932; Sobelman et al. 1995), so that the coherence of two-photon and possibly multi-photon transitions is maintained at least for the lower shells. Here we consider the simplest extension to the classical treatment of the multi-photon cascade and focus only on the two-photon process.

Beginning with the paper of Göppert-Mayer (1931), several textbooks of quantum electrodynamics (Akhiezer \& Berestetskii 1965; Berestetskii et al. 1982) discuss the two-photon emission process. Using the formulation of quantum-electrodynamics, one naturally obtains a nearly Lorentzian shape of the line profiles in the vicinity of the resonances, which also allows us to check for tiny deviations in the real two-photon emission from the spectrum obtained using the simplest classical cascade treatment. As we discuss below, at least in the decay of high s and d-states quantum-electrodynamical corrections lead to additional broad continuum emission and strong deviations of the profiles from the natural Lorentzian shape in the very

\footnotetext{
2 We restrict ourselves to the non-relativistic formulation of the hydrogen atom and assume that it is at rest in the lab frame.

3 More rigorously, collisions more likely destroy the coherence for photons emitted very close to the line center, since in the distant wings the typical characteristic time of processes involving the corresponding quantum-states is much shorter (e.g. Karshenboim \& Ivanov 2007).
}

distant wings of the resonant lines. In this paper we investigate up to what distance the wings of the two-photon emission spectrum in the vicinity of the Lyman- $\alpha$ line continue to have a Lorentzian shape. These deviations in the red wings are the reason for the corrections to the hydrogen recombination history due to the two-photon transitions from high s and d-states. Similarly, these modifications of the Lyman- $\alpha$ line profile could also be important during the initial stages of reionization in the low $-z$ Universe. One should mention that the developed picture is valid for the primordial chemical composition of the Universe, which is characterized by a complete absence of heavy elements, e.g. dust and low ionization energy species that would influence the escape of Lyman- $\alpha$ photons in planetary nebulae and $\mathrm{H}$ II regions in present-day galaxies.

For hydrogen, several publications on the theoretical value of the total $2 \mathrm{~s} \rightarrow 1 \mathrm{~s}$ two-photon decay rate can be found (Breit \& Teller 1940; Kipper 1950; Spitzer \& Greenstein 1951; Klarsfeld 1969; Johnson 1972; Goldman \& Drake 1981; Drake 1986; Goldman 1989) with recent computations performed by Labzowsky et al. (2005) yielding $A_{2 \mathrm{~s} 1 \mathrm{~s}}=8.2206 \mathrm{~s}^{-1}$. In these calculations one has to consider all the possible intermediate states (bound and continuum) with angular momentum quantum number $l=1$, i.e. p-states. Within the non-relativistic treatment of the hydrogen atom for the metastable 2s-level, no p-state with energy lower than the 2s-state exists; hence the twophoton process only involves transitions via virtual intermediate states, without any resonant contributions. Therefore the total two-photon decay rate of the 2 s-level is very low and the 2 s-state has an extremely long lifetime $(\sim 0.12 \mathrm{~s})$. We show that the formulae obtained by Cresser et al. (1986) are not applicable in this case (see Sect. 4.1).

Also, some calculations for the $3 \mathrm{~s}$ and $3 \mathrm{~d}$ two-photon transitions to the ground state have been carried out (Quattropani et al. 1982; Tung et al. 1984; Florescu 1984), but here a problem arises in connection with the contribution from the intermediate $2 \mathrm{p}$-state, which has an energy below the initial level. The corresponding term is dominating the total two-photon decay probability for the $3 \mathrm{~s}$ and $3 \mathrm{~d}$ two-photon process and is connected with the resonant transition via an energetically lower level. It can be interpreted as a cascade involving the quasi-simultaneous emission of two-photons. As in the case of the 2s-level, a broad continuum emission also appears due to transitions via virtual intermediate states, with energies above the initial level. This continuum is not connected to any resonances and therefore has a much lower amplitude. In addition to the cascade-term and this non-resonant term, an interference-term also appears for which a clear interpretation is difficult within the classical formulation. Similarly, in the two-photon decay process of higher $n$ s and $n \mathrm{~d}-$ states to the ground state, $(2 n-4)$-resonances appear, yielding complex structures in the distribution of emitted photons. Some additional examples of $2 \gamma$ emission spectra can also be found in Quattropani et al. (1982) and Tung et al. (1984).

As mentioned above, astrophysicists usually interpret the two-photon cascade as a $1+1$-single photon process. Since even in the full two-photon formulation, the cascade-term dominates the total two-photon decay rate (hence defining the lifetime of the initial $n$ s and $n$ d-states), in a vacuum the total two-photon decay rate should be very close to the $1+1$-single photon rate of the considered level. In the $1+1$-photon picture, the spontaneous two-photon decay rate is simply given by the sum of all spontaneous one-photon decay rates from the initial state, since after the detection of one photon, say a Balmer- $\alpha$ photon in the $3 \mathrm{~s} \rightarrow 1 \mathrm{~s}$ transition, in vacuum the presence of a Lyman- $\alpha$ photon is certain and therefore should not affect the total 3 s decay 
probability. For the 3s and 3d-states Florescu (1984) computed the total spontaneous two-photon decay rate and indeed found $A_{3 \mathrm{~s} \rightarrow 1 \mathrm{~s}}^{2 \gamma} \sim A_{3 \mathrm{~s} \rightarrow 2 \mathrm{p}}^{1 \gamma} \approx 6.317 \times 10^{6} \mathrm{~s}^{-1}$ and $A_{3 \mathrm{~d} \rightarrow 1 \mathrm{~s}}^{2 \gamma} \sim A_{3 \mathrm{~d} \rightarrow 2 \mathrm{p}}^{1 \gamma} \approx$ $6.469 \times 10^{7} \mathrm{~s}^{-1}$. This also suggests that, very close to the resonances, the $1+1$-photon description provides a viable approximation, in which the line profile is very close to a Lorentzian. However, as we show below, quantum-electrodynamical corrections (e.g. virtual intermediate states, interference, correlations of the photons in energy) lead to differences in the two-photon profiles in comparison with the 1+1-single photon profile, which are significant especially in the distant wings, far from the resonances. In particular the interference term plays a crucial role in this context and cannot be neglected.

\section{Two-photon transitions of the $n s$ and $n d-s t a t e s$}

Considering only cases when the initial states is either an $n \mathrm{~s}$ or $n$ d-level and the final state corresponds to a s-level, one can simplify the general formula for the two-photon transition probability as given by Göppert-Mayer (1931) considerably. First, the average over the directions and polarizations of the emitted photons can be carried out immediately, since within the nonrelativistic formulation, one can separate the radial and angular parts of the wave function. For s $\rightarrow$ s-transition, this leads to a global factor of $1 / 27$, while this average yields $2 / 135$ for $\mathrm{d} \rightarrow$ s-transitions (see Tung et al. 1984). Afterwards, the probability for the decay $n_{i} l_{i} \rightarrow 1 \mathrm{~s}$ (where $l_{i}=0$ or $l_{i}=2$ ) with the emission of two photons can be written in terms of the integrals $\left\langle R_{n^{\prime} l^{\prime}}|r| R_{n l}\right\rangle=\left\langle R_{n^{\prime} l^{\prime}}|r| R_{n l}\right\rangle^{*}=\int_{0}^{\infty} R_{n^{\prime} l^{\prime}} r^{3} R_{n l} \mathrm{~d} r$ over the normalized radial functions, $R_{n l}(r)$, for which explicit expressions can be found in the literature (e.g. Sect. 52 in Berestetskii et al. 1982). Then the probability of emitting one photon at frequency $v$ and another at $v^{\prime}$ in the transition $\left(n_{i} l_{i}\right) \rightarrow 1$ s is given by

$$
\begin{aligned}
& \mathrm{d} W_{n_{i} l_{i} \rightarrow 1 \mathrm{~s}}=C_{l_{i}} v^{3} v^{\prime 3}|M|^{2} \mathrm{~d} v \\
& M=\sum_{n=2}\left\langle R_{1 \mathrm{~s}}|r| R_{n \mathrm{p}}\right\rangle\left\langle R_{n \mathrm{p}}|r| R_{n_{i} l_{i}}\right\rangle g_{n}(v) \\
& g_{n}(v)=\frac{1}{E_{n}-E_{n_{i}}+h v}+\frac{1}{E_{n}-E_{n_{i}}+h v^{\prime}} .
\end{aligned}
$$

Here $C_{l}=9 \alpha^{6} c R_{\mathrm{H}} / 2^{10} \times a_{l} / v_{2 \mathrm{~s} 1 \mathrm{~s}}^{5} \approx 4.3663 \mathrm{~s}^{-1} \times a_{l} / v_{2 \mathrm{~s} 1 \mathrm{~s}}^{5}$, with $a_{0}=1$ and $a_{2}=2 / 5$, and where $\alpha$ is the fine structure constant, $c$ the speed of light, $v_{2 \mathrm{~s} 1 \mathrm{~s}}$ the $2 \mathrm{~s}-1 \mathrm{~s}$ transition frequency, and $R_{\mathrm{H}}$ the Rydberg constant for hydrogen. Due to energy conservation, the frequencies of the emitted photons are related by $v+v^{\prime}=v_{i 1 \mathrm{~s}}$, where here $v_{i 1 \mathrm{~s}}$ is the transition frequency between the initial level $n_{i} l_{i}$ and final $1 \mathrm{~s}$-state. The energy of level $n$ is given by $E_{n}=-E_{1 \mathrm{~s}} / n^{2}$, with the $1 \mathrm{~s}$-ionization energy of the hydrogen atom $E_{1 \mathrm{~s}} \approx 13.6 \mathrm{eV}$. The sum in Eq. (1) has to be extended by an integral over the continuum states, but for convenience we omit these in our notation.

For $n_{i}>2$ and $n<n_{i}$, it is clear from Eq. (1) that at $h v=E_{n_{i}}-E_{n}$ and $h v=E_{n}-E_{1 \mathrm{~s}}$, i.e. corresponding to the resonance frequencies to energetically lower levels, one of the denominators inside the sum vanishes, leading to a divergence of the expression. As we discuss below (Sect. 2.1.3), including the lifetime of the intermediate states provides a possibility of removing these singularities (Low 1952; Labzowsky \& Shonin 2004); however, a consistent consideration of this problem requires a more sophisticated treatment beyond the scope of this paper. Physically, transitions to intermediate states with energies
$E_{n} \geq E_{n_{i}}$ are virtual ${ }^{4}$. We split up the sum over all the intermediate states like ${ }^{5} \sum=\sum_{\mathrm{V}}+\sum_{\mathrm{r}}$, where $\sum_{\mathrm{V}}=\sum_{E_{n} \geq E_{n_{i}}}$ and $\sum_{\mathrm{r}}=\sum_{E_{n}<E_{n_{i}}}$ denote the sum over virtual and real intermediate states, respectively. Then we can write

$$
|M|^{2} \propto\left|\sum_{\mathrm{r}}\right|^{2}+\left|\sum_{\mathrm{v}}\right|^{2}+\sum_{\mathrm{r}}^{*} \sum_{\mathrm{v}}+\sum_{\mathrm{r}} \sum_{\mathrm{v}}^{*}
$$

Henceforth, we refer to the contribution to the transition matrix element from $\left|\sum_{\mathrm{r}}\right|^{2}$ as the cascade part, due to $\left|\sum_{\mathrm{v}}\right|^{2}$ as nonresonant part, and the rest as the interference ${ }^{6}$ part. This distinction is ad hoc and not unique, but only motivated by our separation of the infinite sum, which will turn out to be fairly convenient in terms of evaluation of the spectrum and total twophoton decay rate.

The non-resonant contribution to the two-photon decay probability, $\mathrm{d} W^{\mathrm{nr}}$, is then given by

$$
\begin{aligned}
& \mathrm{d} W_{n_{i} l_{i} \rightarrow 1 \mathrm{~s}}^{\mathrm{nr}}=C_{l_{i}} v^{3} v^{\prime 3}\left|M_{\mathrm{nr}}\right|^{2} \mathrm{~d} v \\
& M_{\mathrm{nr}}=\sum_{n=n_{i}}^{\infty}\left\langle R_{1 \mathrm{~s}}|r| R_{n \mathrm{p}}\right\rangle\left\langle R_{n \mathrm{p}}|r| R_{n_{i} l_{i}}\right\rangle g_{n}(v) .
\end{aligned}
$$

For the cascade and interference terms, one instead has

$$
\begin{aligned}
& \mathrm{d} W_{n_{i} l_{i} \rightarrow 1 \mathrm{~s}}^{\mathrm{cas}}=C_{l_{i}} v^{3} v^{\prime 3}\left|M_{\mathrm{cas}}\right|^{2} \mathrm{~d} v \\
& M_{\text {cas }}=\sum_{n=2}^{n_{i}-1}\left\langle R_{1 \mathrm{~s}}|r| R_{n \mathrm{p}}\right\rangle\left\langle R_{n \mathrm{p}}|r| R_{n_{i} l_{i}}\right\rangle g_{n}(v)
\end{aligned}
$$

and

$$
\mathrm{d} W_{n_{i} l_{i} \rightarrow 1 \mathrm{~s}}^{\text {int }}=C_{l_{i}} v^{3} v^{\prime 3}\left[M_{\mathrm{cas}}^{*} M_{\mathrm{nr}}+M_{\mathrm{cas}} M_{\mathrm{nr}}^{*}\right] \mathrm{d} v,
$$

respectively.

\subsection{Total two-photon decay rate}

In order to obtain the total two-photon decay rate in vacuum one now has to integrate Eq. (1) over all possible frequencies $v$. The corresponding integral can be cast into the form

$$
A_{n_{i} l_{i} \rightarrow 1 \mathrm{~s}}^{2 \gamma}=\frac{1}{2} \int_{0}^{v_{i 1 \mathrm{~s}}} \mathrm{~d} W_{n_{i} l_{i} \rightarrow 1 \mathrm{~s}}=\frac{1}{2} \int_{0}^{1} \phi_{n_{i} l_{i} \rightarrow 1 \mathrm{~s}}^{2 \gamma}(y) \mathrm{d} y,
$$

with $y=v / v_{i 1 \mathrm{~s}}$ and where the factor of $1 / 2$ avoids doublecounting of photons. In Eq. (6) we have introduced the twophoton decay profile function,

$\phi_{n_{i} l_{i} \rightarrow 1 \mathrm{~s}}^{2 \gamma}(y)=\phi_{n_{i} l_{i} \rightarrow 1 \mathrm{~s}}^{\mathrm{nr}}(y)+\phi_{n_{i} l_{i} \rightarrow 1 \mathrm{~s}}^{\mathrm{cas}}(y)+\phi_{n_{i} l_{i} \rightarrow 1 \mathrm{~s}}^{\mathrm{int}}(y)$,

which is the sum of the two-photon profiles due to the nonresonant, cascade, and interference terms, respectively. Below we discuss the contribution of each term separately.

\footnotetext{
${ }^{4}$ Strictly speaking, photons appearing in the distant wings of the resonance are also connected with virtual states (e.g. Karshenboim \& Ivanov 2007).

5 Within the non-relativistic treatment of the hydrogen atom, this is equivalent to separating the cases $n<n_{i}$ and $n \geq n_{i}$. Since the energy of the continuum states is always greater than for the bound states, the former are associated with the case $n \geq n_{i}$.

${ }^{6}$ In principle one should be more accurate by calling this contribution resonant/non-resonant-interference term, since also some level of interference is already included inside the resonant and non-resonant-term, which is absent in the 1+1-photon picture. However, we generally do not make this distinction.
} 


\subsubsection{Interpretation of the two-photon emission profile}

Physically the two-photon emission profile or spectrum $\phi_{n_{i} l_{i} \rightarrow 1 \mathrm{~s}}^{2 \gamma}$ defines the number of photons that are released per second in the frequency interval between $v$ and $v+\mathrm{d} v$. If one integrates over the whole spectrum, this therefore yields the total number of photons emitted per second due to the two-photon transition. The two-photon profile includes both photons at the same time, so that the total two-photon transition rate per initial s or d-state has to be divided by a factor of 2 (see Eq. (6)).

Due to energy conservation it is clear that, when detecting a photon that was produced in a particular two-photon transition from some initial $n$ s or $n d$-state to the ground state, at a frequency $v$, the other photon has frequency $v^{\prime}=v_{n 1 \mathrm{~s}}-v$. Therefore also the probability to release a photon at $v$ should be equal to the probability for the emission of a photon at $v_{n 1 \mathrm{~s}}-v$, a property that is reflected in the symmetry of the two-photon profiles around $y=1 / 2$ (see Sect. 3 for more explicit examples).

\subsubsection{Total two-photon decay rate due to the non-resonant contribution}

In Eq. (7) we have introduced the non-resonant two-photon decay profile function, $\phi_{n_{i} l_{i} \rightarrow 1 \mathrm{~s}}^{\mathrm{nr}}(y)$, which can be written as

$$
\begin{aligned}
& \phi_{n_{i} l_{i} \rightarrow 1 \mathrm{~s}}^{\mathrm{nr}}(y)=G_{n_{i} l_{i}} y^{3}(1-y)^{3}\left|\tilde{M}_{\mathrm{nr}}\right|^{2} \\
& \tilde{M}_{\mathrm{nr}}=\sum_{n=n_{i}}^{\infty}\left\langle R_{1 \mathrm{~s}}|r| R_{n \mathrm{p}}\right\rangle\left\langle R_{n \mathrm{p}}|r| R_{n_{i} l_{i}}\right\rangle f_{n}(y) \\
& f_{n}(y)=\frac{1}{y+y_{n}^{\mathrm{nr},+}}-\frac{1}{y-y_{n}^{\mathrm{nr},-}}
\end{aligned}
$$

with $G_{n_{i} l_{i}}=v_{i 1 \mathrm{~s}}^{5} C_{l_{i}}$. Here we defined the frequencies

$y_{n}^{\mathrm{nr},+}=\frac{n^{2}-n_{i}^{2}}{n^{2}\left(n_{i}^{2}-1\right)}$

$y_{n}^{\mathrm{nr},-}=1+y_{n}^{\mathrm{nr},+}=\frac{n_{i}^{2}\left(n^{2}-1\right)}{n^{2}\left(n_{i}^{2}-1\right)}$,

which for $n \geq n_{i}$ vary within the ranges $0 \leq y_{n}^{\mathrm{nr},+} \leq 1 /\left(n_{i}^{2}-1\right)$ and $1 \leq y_{n}^{\mathrm{nr},+} \leq n_{i}^{2} /\left(n_{i}^{2}-1\right)$. For $n=n_{i}$ one finds $y_{n}^{\mathrm{nr},+}=0$ and $y_{n}^{\mathrm{nr},-}=1$. Since the sum in $\tilde{M}_{\mathrm{nr}}$ only involves intermediate states with $n \geq n_{i}$, the denominators of $f_{n}$ never vanish within the interval $0<y<1$, and for $n=n_{i}$ the factors $y^{3}$ and $(1-y)^{3}$ ensure that $\tilde{M}_{\mathrm{nr}}$ approaches zero within the limits $y \rightarrow 0$ and $y \rightarrow 1$. In addition $\tilde{M}_{\mathrm{nr}}$ is real and symmetric around $y=1 / 2$.

To compute the total rate one now only has to replace $\phi_{n_{i} l_{i} \rightarrow 1 \mathrm{~s}}^{2 \gamma}(y)$ in Eq. (6), by the expression (8). To evaluate the sum and integrals over the radial functions, we used MATHEMATICA. Normally we restrict ourselves to the first 200 terms in the sum, but computations with up to 4000 terms were also performed for the $2 \mathrm{~s}, 3 \mathrm{~s}$, and $3 \mathrm{~d}$ rates. Within the assumptions the results for the other levels should be correct to better than $\sim 1 \%$. To make cross checks easier, we give the expression for the necessary radial integrals $\left\langle R_{n^{\prime} l^{\prime}}|r| R_{n l}\right\rangle$ up to $n_{i}=5$ in Appendix A.

\subsubsection{Total two-photon decay rate due to the cascade and interference terms}

For the cascade and interference terms special care has to be taken close to and at intermediate distances from the resonance frequencies $h v=E_{n_{i}}-E_{n}$ and $h v=E_{n}-E_{1 \mathrm{~s}}$. As mentioned above, a consistent treatment of this problem requires more sophisticated methods, including the amplitudes of several additional processes (e.g. Karshenboim \& Ivanov 2007), than are within the scope of this paper. One simple approximate solution to this problem can be given when taking the lifetime of the intermediate states into account as a small imaginary contribution to their energy ${ }^{7}$. Including this shift into the equations for the $2 p$-transition leads to the classical expression of the Lorentzian within the formulation of Quantum Electrodynamics and can be attributed to the first order radiative corrections of the one-photon process (Low 1952; Labzowsky \& Shonin 2004). Florescu (1984) used this approach to compute the total 3s and $3 \mathrm{~d}$ two-photon decay rates and simply replaced the energy, $E_{2 \mathrm{p}}$, of the $2 p$-state by, $E_{2 p}-\mathrm{i} h \Gamma_{2 p} / 2$, where $\Gamma_{2 p}$ is the width of the $2 \mathrm{p}$-state due to spontaneous transitions. Except for the 2 s-state, summing all the one-photon decay rates (e.g. these values can be computed using the routines of Storey \& Hummer 1991) should yield a very good approximation for the total lifetime of any given initial level in the hydrogen atom. For estimates, we therefore follow this approximate procedure and replace the energies of all intermediate p-states by $E_{n \mathrm{p}} \rightarrow E_{n \mathrm{p}}-\mathrm{i} h \Gamma_{n \mathrm{p}}^{1 \gamma} / 2$. Here $\Gamma_{n \mathrm{p}}^{1 \gamma} \equiv A_{n \mathrm{p}}^{1 \gamma}=\sum_{n^{\prime} l^{\prime}} A_{n \mathrm{p} \rightarrow n^{\prime} l^{\prime}}^{1 \gamma}$ is the total $1 \gamma$-width of the intermediate $n \mathrm{p}$-state due to spontaneous transitions. Note that for $n>2$ the p-states can decay via channels, which do not directly lead to the $1 \mathrm{~s}$-state, thereby leading to the possible emission of more than two photons.

The two-photon contribution to the total lifetime of the s and d-levels for transitions to the ground state should be close to the value following from the sum of the rates for all possible $1 \gamma$-transitions to lower-lying intermediate p-states multiplied by the probability that the electron will "afterwards" go directly to the $1 \mathrm{~s}-$ level $^{8}$ :

$\Gamma_{n \mathrm{~s} / \mathrm{d}}^{2 \gamma} \approx \sum_{n^{\prime}=2}^{n-1} A_{n \mathrm{~s} / \mathrm{d} \rightarrow n^{\prime} \mathrm{p}}^{1 \gamma} \times \frac{A_{n^{\prime} \mathrm{p} \rightarrow 1 \mathrm{~s}}^{1 \gamma}}{A_{n^{\prime} \mathrm{p}}^{1 \gamma}}$,

where $A_{n \mathrm{~s} / \mathrm{d} \rightarrow n^{\prime} l^{\prime}}^{1 \gamma}$ is the spontaneous one-photon Einstein coefficients of the considered transition from the initial s/d-state ${ }^{9}$. Although for the s-levels, this already accounts for all the possible one-photon decay channels, d-states with $n>4$ can also decay via intermediate f-states. However, in this case again more than two-photons have to be released in order to reach the $1 \mathrm{~s}-$ level and therefore do not contribute anything here. The addition (9) to the energies is not important for the non-resonant term, but it becomes crucial very close to the resonances for both the cascade and interference term. However, here we are mainly interested in the behavior at large distances from the poles $\left(|\Delta v| \gg \Gamma_{n \mathrm{p}}^{1 \gamma}\right)$, so that this replacement has no direct effect on the discussed cosmological application (see Sect. 5.3).

Here one may ask, why the lifetime of the initial level is not included? Physically this is motivated by the idea that, following the interpretation of Weisskopf \& Wigner (1930), we consider one particular initial "energy sub-level" and do not specify the process that populated it. Therefore the final profile should be independent of the shape of the distribution of energy-sublevels around the mean energy of the initial state. One can also

\footnotetext{
7 We neglect the small correction to the real part of the energy caused by the Lamb-shift.

8 The authors wish to thank E.E. Kholupenko for pointing out some inaccuracies related to this aspect. However, the modification did not affect the results of this paper.

9 Here $A_{n s / \mathrm{d} \rightarrow n^{\prime} l^{\prime}}^{1 \gamma}$ refers to either the $A_{n s \rightarrow n^{\prime} l^{\prime}}^{1 \gamma}$ or the $A_{n \mathrm{~d} \rightarrow n^{\prime} l^{\prime}}^{1 \gamma}$ decay rate. We use this notation more often below.
} 
consider this as equivalent to neglecting any possible reshuffling of the electron by perturbing particles while it is in the initial state. However, in the computation presented below we do not approach the resonances so close that these differences would play any role.

With the notation of Sect. 2.1.2, we now introduce the cascade and interference two-photon emission profiles by

$\phi_{n_{i} l_{i} \rightarrow 1 \mathrm{~s}}^{\text {cas }}(y)=G_{n_{i} l_{i}} y^{3}(1-y)^{3}\left|\tilde{M}_{\mathrm{cas}}\right|^{2}$

$\phi_{n_{i} l_{i} \rightarrow 1 \mathrm{~s}}^{\text {int }}(y)=G_{n_{i} l_{i}} y^{3}(1-y)^{3} \tilde{M}_{\mathrm{nr}}\left[\tilde{M}_{\text {cas }}^{*}+\tilde{M}_{\text {cas }}\right]$,

where the cascade matrix element is given by

$\tilde{M}_{\mathrm{cas}}=\sum_{n=2}^{n_{i}-1}\left\langle R_{1 \mathrm{~s}}|r| R_{n \mathrm{p}}\right\rangle\left\langle R_{n \mathrm{p}}|r| R_{n_{i} l_{i}}\right\rangle h_{n}(y)$

$h_{n}(y)=\frac{1}{y-y_{n}^{\mathrm{cas},+}-i \delta_{n}}-\frac{1}{y-y_{n}^{\mathrm{cas},-}-i \delta_{n}}$.

Here $\delta_{n}=\Gamma_{n \mathrm{p}} / 4 \pi v_{i 1 \mathrm{~s}}$ accounts for the energy-shifts due to the finite lifetime of the intermediate $n$ p-state. We have also introduced the resonance frequencies

$y_{n}^{\mathrm{cas},+} \equiv-y_{n}^{\mathrm{nr},+}$

$y_{n}^{\mathrm{cas},-} \equiv y_{n}^{\mathrm{nr},-}$.

Since for the cascade and interference term $n<n_{i}$, these now have values strictly within the range $0<y<1$.

Defining the function

$L(a, b)=\frac{a}{a^{2}+b^{2}}$,

which actually has the generic shape of a Lorentzian, then $f_{n}(y)=f_{n}^{\mathrm{r}}(y)+\mathrm{i} f_{n}^{\mathrm{i}}(y)$, where the real and imaginary part of $f_{n}$ are defined by

$f_{n}^{\mathrm{r}}(y)=L\left(y-y_{n}^{\mathrm{cas},+}, \delta_{n}\right)-L\left(y-y_{n}^{\mathrm{cas},-}, \delta_{n}\right)$

$f_{n}^{\mathrm{i}}(y)=L\left(\delta_{n}, y-y_{n}^{\mathrm{cas},+}\right)-L\left(\delta_{n}, y-y_{n}^{\mathrm{cas},-}\right)$,

respectively. Introducing $\kappa_{n}=\left\langle R_{1 \mathrm{~s}}|r| R_{n \mathrm{p}}\right\rangle\left\langle R_{n \mathrm{p}}|r| R_{n_{i} l_{i}}\right\rangle$ one can rewrite $\left|\tilde{M}_{\text {cas }}\right|^{2}$ and $\left|\tilde{M}_{\text {int }}\right|^{2}=\tilde{M}_{\mathrm{nr}}\left[\tilde{M}_{\text {cas }}^{*}+\tilde{M}_{\text {cas }}\right]$ as

$\left|\tilde{M}_{\text {cas }}\right|^{2}=\sum_{n=2}^{n_{i}-1} \kappa_{n}^{2}\left|f_{n}(y)\right|^{2}+2 \sum_{n=2}^{n_{i}-1} \sum_{m=2}^{n-1} \kappa_{n} \kappa_{m}\left[f_{n}^{\mathrm{r}} f_{m}^{\mathrm{r}}+f_{n}^{\mathrm{i}} f_{m}^{\mathrm{i}}\right]$

$\left|\tilde{M}_{\mathrm{int}}\right|^{2}=2 \tilde{M}_{\mathrm{nr}} \times \sum_{n=2}^{n_{i}-1} \kappa_{n} f_{n}^{\mathrm{r}}(y)$.

To compute the total rate one now only has to replace $\phi_{n_{i} l_{i} \rightarrow 1 \mathrm{~s}}^{2 \gamma}(y)$ in Eq. (6), by the corresponding expressions (10).

\section{Two-photon emission spectra}

\subsection{Non-resonant two-photon emission spectra}

In Fig. 1 we present the profile functions for the non-resonant contribution to the two-photon decay spectrum. All the profiles have a maximum at $y=1 / 2$. For the $n \mathrm{~s} \rightarrow 1 \mathrm{~s}$-emission profiles the difference in the shape of the curves is quite big, while for initial d-states in general the profile does not vary as much. However, in both cases the amplitude at $y=1 / 2$ changes strongly, increasing towards larger $n$ (see Appendix B).

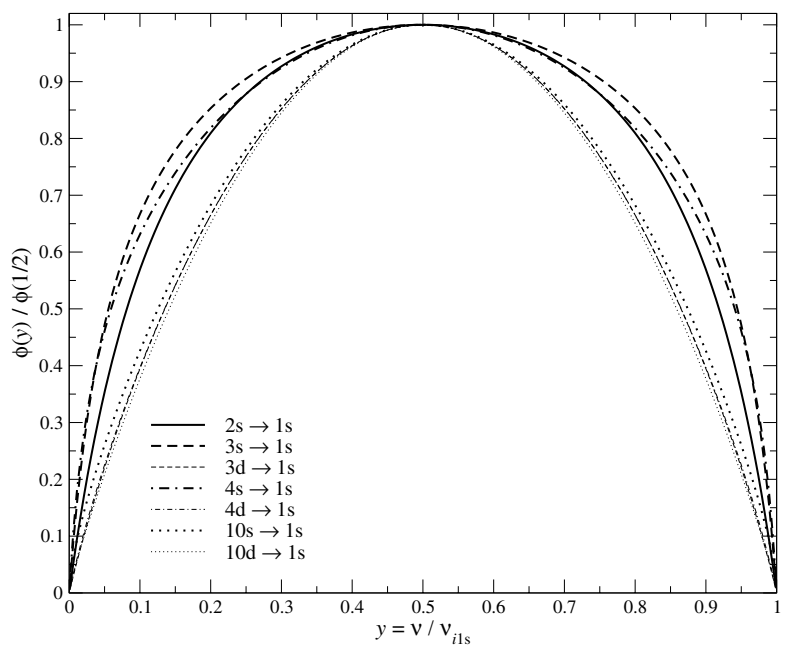

Fig. 1. Non-resonant two-photon emission spectra, Eq. (8), for several transitions. All curves are normalized to unity at $y=1 / 2$. The values of $\phi^{\mathrm{nr}}(1 / 2)$ can be found in Appendix B.

Due to our separation of the infinite sum over the intermediate substates, the sums in the cascade and interference terms become finite. This allows us to evaluate $M_{\mathrm{nr}}$ numerically and use convenient fitting formulae for their representation. Realizing that $M_{\mathrm{nr}}$ is symmetric around $y=1 / 2$ and that it scales like $\sim 1 / y$ and $\sim 1 /(1-y)$ at the boundaries, we approximated $y(1-y) M_{\mathrm{nr}}$. In Appendix B the obtained formulae for all $n \mathrm{~s} \rightarrow 1 \mathrm{~s}$ and $n \mathrm{~d} \rightarrow 1 \mathrm{~s}$ transitions up to $n=20$ are given. For the non-resonant term within the range $10^{-3} \leq y \leq 0.999$, these approximations should be accurate to better than $0.1 \%$. Since these are fast and simple to evaluate they should be useful for analytic estimates and numerical applications. Note that all non-resonant matrixelements are negative.

\subsection{The $3 s$ and $3 d$ two-photon emission spectrum}

In Fig. 2 we give the full two-photon emission spectra for the $3 \mathrm{~s} \rightarrow 1 \mathrm{~s}$ and $3 \mathrm{~d} \rightarrow 1 \mathrm{~s}$ transition. In both cases, the non-resonance term increases the wings of the profiles close to $y \sim 0$ and $y \sim 1$. However, the interference between the non-resonant and cascade part is destructive in the central region $(y \sim 1 / 2)$ and significantly reduces the amplitude of the total two-photon emission. It even leads to full cancellation at $y \sim 0.22$ and $y \sim 0.78$ for the $3 \mathrm{~s}-$ level, whereas for the $3 \mathrm{~d}$-level the photon production does not vanish in the region between the resonances (see also Tung et al. 1984).

For both the $3 \mathrm{~s} \rightarrow 1 \mathrm{~s}$ and $3 \mathrm{~d} \rightarrow 1 \mathrm{~s}$ transitions, only one term in the cascade appears, which is related to the transition via the intermediate $2 \mathrm{p}$-state. The matrix elements for these are $\kappa_{2}^{3 \mathrm{~s}} \approx 1.211$ and $\kappa_{2}^{3 \mathrm{~d}} \approx 6.126$, and according to Eq. (11) the resonance frequencies are at $y_{2}^{\text {cas, }+}=5 / 32$ (Balmer- $\alpha$ transition) and $y_{2}^{\text {cas, }-}=27 / 32$ (Lyman- $\alpha$ transition). With the equations given in Sect. 2.1.3 and using the fitting formulae according to Appendix B one can analytically approximate the full twophoton emission spectrum. As Fig. 2 shows the agreement is excellent at all considered frequencies.

\subsection{The ns and nd two-photon emission spectrum}

As an example, in Fig. 3 we present the full two-photon emission spectra for the $5 \mathrm{~s} \rightarrow 1 \mathrm{~s}$ and $5 \mathrm{~d} \rightarrow 1 \mathrm{~s}$ transition. Again one can 

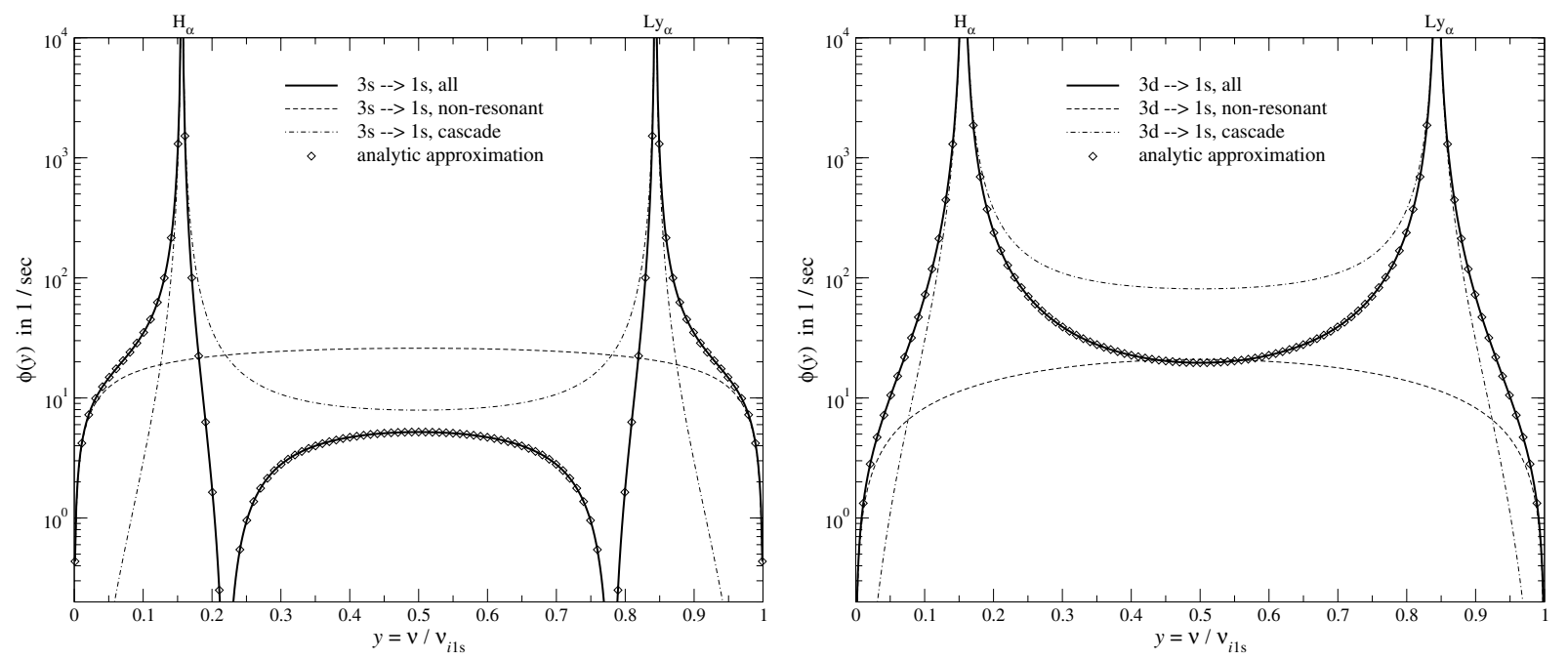

Fig. 2. Two-photon emission spectra for the $3 \mathrm{~s} \rightarrow 1 \mathrm{~s}$ and $3 \mathrm{~d} \rightarrow 1 \mathrm{~s}$ transitions. The non-resonant, cascade, and combined spectra are shown as labeled. Also we give the analytic approximation based on the equations discussed in Sect. 2.1.3, together with the fitting formula for the $2 \mathrm{~s}$ and $3 \mathrm{~d}$ non-resonant matrix element (see Appendix B). The resonances correspond to the Balmer- $\alpha$ and Lyman- $\alpha$ transitions.

see that the interference term strongly affects the shape of the spectrum in the wings of the resonances. In particular, destructive interference close to $y=1 / 2$ strongly reduces the total amplitude of the emission. For the 5s-level, interference leads to full cancellation of the photon production $(y \sim 0.28$ and $y \sim 0.72)$ in the region between the innermost resonances, whereas the photon production does not vanish within this range for the 5d-level. This difference is characteristic of the shape of the s and d-twophoton spectra, also for higher values of $n$. It is also clear, that using the equations given in Sect. 2.1.3, together with the fitting formulae according to Appendix B, one can analytically approximate the full two-photon emission spectrum with very high accuracy in the full range of considered frequencies.

For initial states with a higher value of $n_{i}$, more resonances (in total $2 n_{i}-4$ ) appear, but otherwise the spectra look very similar and do not add any deeper physical aspects. We checked the analytic approximations for the full two-photon emission spectrum of several $n \mathrm{~s} \rightarrow 1 \mathrm{~s}$ and $n \mathrm{~d} \rightarrow 1$ s two-photon transition up to $n=20$ and always found excellent agreement with the results from our full numerical treatment.

\section{Total two-photon decay rates}

\subsection{The two-photon rate for the 2 s-state}

It is clear that, within the non-relativistic formulation for the $2 s \rightarrow 1$ s-two-photon transition of hydrogen-like ions, only nonresonant contributions to the total lifetime exist. When we use Eq. (6) together with Eq. (8) and include the first 4000 terms in the infinite sum, we obtain the value $A_{2 \mathrm{~s} 1 \mathrm{~s}}=8.2293 \mathrm{~s}^{-1}$, which fully agrees with the result of earlier computations (Breit \& Teller 1940; Kipper 1950; Spitzer \& Greenstein 1951; Klarsfeld 1969; Johnson 1972; Goldman \& Drake 1981; Drake 1986; Goldman 1989; Labzowsky et al. 2005). With the approximate formula for the non-resonant two-photon emission spectrum for the 2 s-state as given in Appendix $\mathrm{B}$, we obtain $A_{2 \mathrm{~s} 1 \mathrm{~s}}=$ $8.2297 \mathrm{~s}^{-1}$, which shows the high accuracy of the approximation.

Equation (6), together with Eq. (8), is very similar to Eq. (14) in the work of Cresser et al. (1986). The expression of Cresser et al. (1986) was obtained using general arguments about the total lifetime of the considered level, and according to their work it should be applicable to all s and d-states of hydrogen, yielding the two-photon correction to the lifetime. However, if applied to the hydrogen $2 \mathrm{~s}-$ level one finds $A_{2 \mathrm{~s} 1 \mathrm{~s}}^{\mathrm{Cr}}=1.4607 \mathrm{~s}^{-1}$ instead of $A_{2 \mathrm{~s} 1 \mathrm{~s}}=8.2293 \mathrm{~s}^{-1}$. The difference stems from the fact that here, like in the publications mentioned above, we included the term with $n \equiv n_{i}$ in the sum Eq. (8). This shows that the largest contribution to the total 2 s-two-photon decay rate (in this case equivalent to the non-resonant contribution) actually comes from the transition via the intermediate 2 p-state, i.e. the matrix element $\left\langle R_{1 \mathrm{~s}}|r| R_{2 \mathrm{p}}\right\rangle\left\langle R_{2 \mathrm{p}}|r| R_{2 \mathrm{~s}}\right\rangle \approx-6.704$, and cannot be neglected. This suggests that the arguments by Cresser et al. (1986) are incomplete, or at least not generally applicable.

Although to our knowledge only rough direct measurements of the two-photon decay rate exist for the hydrogen 2 s-state (Krüger \& Oed 1975; Cesar et al. 1996), one can find experimental confirmations (Prior 1972; Kocher et al. 1972; Hinds et al. 1978) of the theoretical value for the two-photon decay rate of the hydrogen-like helium ion $\left(\Gamma_{\text {th }} \approx 8.229 Z^{6} \mathrm{~s}^{-1}\right)$, which do reach percent-level accuracy. Also measurement for hydrogen-like Ar, F, and O exist (Marrus \& Schmieder 1972; Cocke et al. 1974; Gould \& Marrus 1983), but with lower accuracy. These experimental confirmations further support the idea that, in theoretical computations of the total two-photon decay rate and in particular the correction to the one-photon lifetime, it is not enough to consider only intermediate states with energies $E_{n}>E_{i}$.

For hydrogen-like ions, care should be taken when computing the 2s-two photon decay rate within the relativistic treatment. In this case the $2 \mathrm{p}_{1 / 2}$ level due to the Lamb-shift and fine-structure splitting energetically lies below the $2 \mathrm{~s}_{1 / 2}$ level. Increasing $Z$ will make this shift even bigger, but as the measurements for He and Ar show, this intermediate state cannot contribute beyond the percent-level to the total lifetime of the corresponding $2 s_{1 / 2}$-state. This is also expected because the lifetime of the $2 \mathrm{~s}$-state should not be strongly altered by the slow $2 \mathrm{~s}_{1 / 2} \rightarrow 2 \mathrm{p}_{1 / 2}$ transition $\left(\sim 1.6 \times 10^{-9} \mathrm{~s}^{-1}\right)$. In addition, the poles due to this intermediate state lie very close to $v \rightarrow 0$ and $v \rightarrow v_{i 1 \mathrm{~s}}$ and are therefore suppressed by the factors of $v^{3} v^{\prime 3}$ in Eq. (1). 

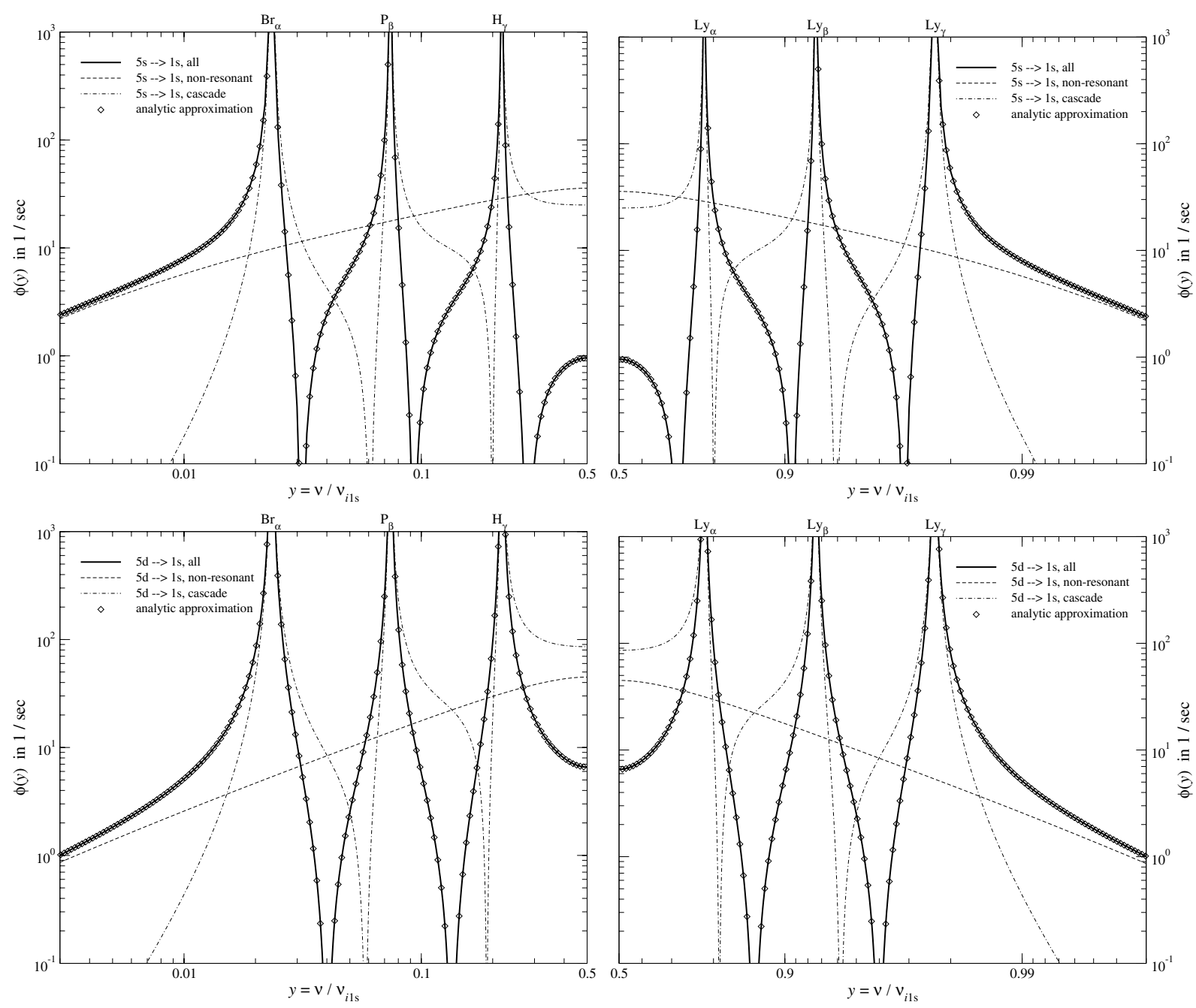

Fig. 3. Two-photon emission spectra for the $5 \mathrm{~s} \rightarrow 1 \mathrm{~s}$ and $5 \mathrm{~d} \rightarrow 1 \mathrm{~s}$ transitions. The non-resonant, cascade, and combined spectra are shown as labeled. Also we give the analytic approximation based on the equations discussed in Sect. 2.1.3, together with the fitting formula for the 5s and $5 \mathrm{~d}$ non-resonant matrix element (see Appendix B). The resonances correspond to the Brackett- $\alpha$, Paschen- $\beta$, and Balmer- $\gamma$ transitions at $y<1 / 2$, and Lyman- $\alpha$, Lyman- $\beta$, and Lyman- $\gamma$ at $y>1 / 2$.

\subsection{The two-photon rates for the $3 s$ and $3 d$-states}

\subsubsection{The non-resonant contribution}

Using the formula given by Cresser et al. (1986), i.e. explicitly neglecting the transition via the intermediate $3 p$-state, we can reproduce their values for the non-resonant contribution to the two-photon decay rates of the $3 \mathrm{~s} \rightarrow 1 \mathrm{~s}$ and $3 \mathrm{~d} \rightarrow 1 \mathrm{~s}$ transitions. Later Florescu et al. (1988) computed these values again within the framework of Cresser et al. (1986) but to higher accuracy. We are also able to reproduce these results $\left(A_{3 \mathrm{~s} \rightarrow 1 \mathrm{sr}}^{\mathrm{nr}, \mathrm{Cr}}=8.22581 \mathrm{~s}^{-1}\right.$ and $A_{3 \mathrm{~d} \rightarrow 1 \mathrm{~s}}^{\mathrm{nr}, \mathrm{Cr}}=0.131814 \mathrm{~s}^{-1}$ ) up to all given figures. Although the discussion in the previous sections has already shown that these values probably have no direct relation to the total corrections in the lifetime of the level due to the two-photon process, we computed them to check our own computational procedure.

However, returning to our definition of the non-resonant twophoton decay rate, the transition via the intermediate $3 \mathrm{p}$-state has to be included. We then obtain $A_{3 \mathrm{~s} \rightarrow 1 \mathrm{~s}}^{\mathrm{nr}}=10.556(10.558) \mathrm{s}^{-1}$ and $A_{3 \mathrm{~d} \rightarrow 1 \mathrm{~s}}^{\mathrm{nr}}=7.1474(7.1475) \mathrm{s}^{-1}$, where the values in parenthesis were computed by integrating our analytic approximation. In particular, for the $3 \mathrm{~d}$-level, this increases the nonresonant contribution to the total two-photon decay rate by a factor of $\sim 54$. If in the sum (8b) we only consider the term $\left\langle R_{1 \mathrm{~s}}|r| R_{3 \mathrm{p}}\right\rangle\left\langle R_{3 \mathrm{p}}|r| R_{3 \mathrm{~d}}\right\rangle f_{3} \approx-5.199 f_{3}$, with the function $f_{3}=$ $y^{-1}+(1-y)^{-1}$ and the integral $\int_{0}^{1} y^{3}(1-y)^{3} f_{3}^{2} \mathrm{~d} y=1 / 6$, then one obtains $A_{3 \mathrm{~d} \rightarrow 1 \mathrm{~s}}^{\mathrm{nr}} \approx 9.199 \mathrm{~s}^{-1}$. This shows that indeed the main contribution to the non-resonant part of 3d-two-photon decay rate comes from the transition via the intermediate $3 \mathrm{p}$-state.

\subsubsection{The cascade and interference terms}

With the formulae given in Sect. 2, it should be possible to compute the total two-photon decay rate of the $3 \mathrm{~s}$ and $3 \mathrm{~d}$-states. Including the lifetime of the intermediate 2 -state as discussed in Sect. 2.1.3, we also computed the total lifetime of the $3 \mathrm{~s}$ and 3d-states, and, in agreement with Florescu (1984), obtained values that were very close to the one expected from the onephoton lifetime. But as mentioned in Sect. 2.1.3, within the simple approximation used to regularize the cascade and interference terms, it is not possible to compute the total correction to the one-photon lifetime, consistent in the considered order of the fine-structure constant $\alpha$. In addition, as we will see in Sect. 5, this is not necessary for our cosmological application. 
Table 1. The non-resonant contribution to the total two-photon rates for the transitions $n_{i} \mathrm{~s} \rightarrow 1 \mathrm{~s}$ and $n_{i} \mathrm{~d} \rightarrow 1 \mathrm{~s}$ up to $n_{i}=20$.

\begin{tabular}{|c|c|c|c|c|c|}
\hline$n_{i}$ & $A_{n_{i} \mathrm{~s} \rightarrow 1 \mathrm{~s}}^{\mathrm{nr}}$ & $A_{n_{i} \mathrm{~d} \rightarrow 1 \mathrm{~s}}^{\mathrm{nr}}$ & $n_{i}$ & $A_{n_{i} \mathrm{~s} \rightarrow 1 \mathrm{~s}}^{\mathrm{nr}}$ & $\overline{A_{n_{i} \mathrm{~d} \rightarrow 1 \mathrm{~s}}^{\mathrm{nr}}}$ \\
\hline 2 & $8.2293 \mathrm{~s}^{-1}$ & - & $\overline{-}$ & . & - \\
\hline 3 & $10.556 \mathrm{~s}^{-1}$ & $7.1474 \mathrm{~s}^{-1}$ & 12 & $34.096 \mathrm{~s}^{-1}$ & $29.687 \mathrm{~s}^{-1}$ \\
\hline 4 & $11.951 \mathrm{~s}^{-1}$ & $11.942 \mathrm{~s}^{-1}$ & 13 & $37.552 \mathrm{~s}^{-1}$ & $31.410 \mathrm{~s}^{-1}$ \\
\hline 5 & $13.741 \mathrm{~s}^{-1}$ & $15.331 \mathrm{~s}^{-1}$ & 14 & $41.076 \mathrm{~s}^{-1}$ & $33.114 \mathrm{~s}^{-1}$ \\
\hline 6 & $15.954 \mathrm{~s}^{-1}$ & $18.004 \mathrm{~s}^{-1}$ & 15 & $44.659 \mathrm{~s}^{-1}$ & $34.801 \mathrm{~s}^{-1}$ \\
\hline 7 & $18.501 \mathrm{~s}^{-1}$ & $20.293 \mathrm{~s}^{-1}$ & 16 & $48.290 \mathrm{~s}^{-1}$ & $36.478 \mathrm{~s}^{-1}$ \\
\hline 8 & $21.301 \mathrm{~s}^{-1}$ & $22.362 \mathrm{~s}^{-1}$ & 17 & $51.964 \mathrm{~s}^{-1}$ & $38.146 \mathrm{~s}^{-1}$ \\
\hline 9 & $24.296 \mathrm{~s}^{-1}$ & $24.296 \mathrm{~s}^{-1}$ & 18 & $55.674 \mathrm{~s}^{-1}$ & $39.806 \mathrm{~s}^{-1}$ \\
\hline 10 & $27.448 \mathrm{~s}^{-1}$ & $26.144 \mathrm{~s}^{-1}$ & 19 & $59.416 \mathrm{~s}^{-1}$ & $41.462 \mathrm{~s}^{-1}$ \\
\hline 11 & $30.722 \mathrm{~s}^{-1}$ & $27.935 \mathrm{~s}^{-1}$ & 20 & $63.185 \mathrm{~s}^{-1}$ & $43.113 \mathrm{~s}^{-1}$ \\
\hline
\end{tabular}

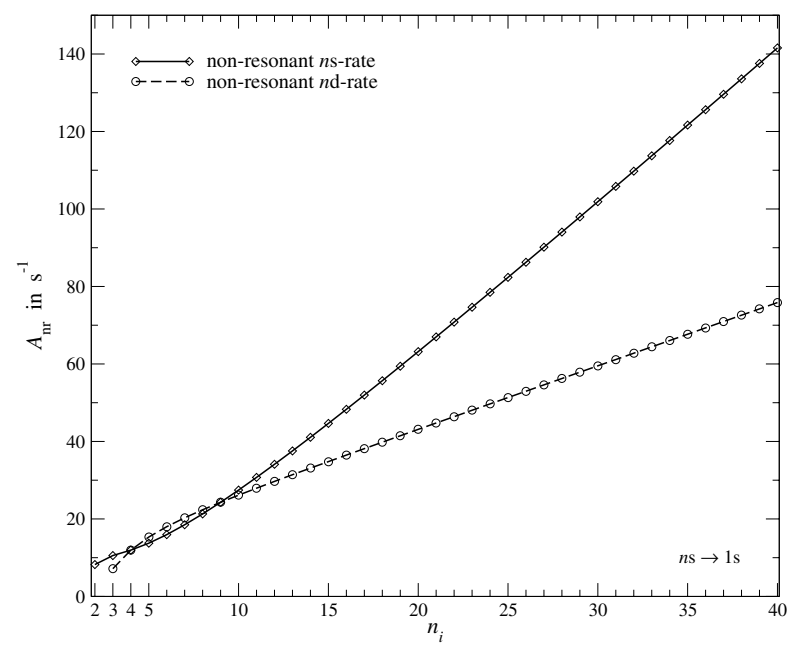

Fig. 4. Non-resonant contribution to the total two-photon decay rate in vacuum for the $n \mathrm{~s}$ and $n \mathrm{~d}$-states of the hydrogen atom. The results were computed using the first 200 terms above $n_{i}$.

However, in order to compare with other computations, it may be useful to give some additional intermediate results. We therefore also integrated the contribution of the interference term separately, yielding $A_{3 \mathrm{~s} \rightarrow 1 \mathrm{~s}}^{\mathrm{int}}=-10.810(-10.810) \mathrm{s}^{-1}$ and $A_{3 \mathrm{~d} \rightarrow 1 \mathrm{~s}}^{\text {int }}=-30.019(-30.019) \mathrm{s}^{-1}$. This shows that, because of interference, the small increase of the decay-rate due to the nonresonant term (see Table 1) is completely canceled, again emphasizing how important the interference term is. In Table 1 we included a maximal number of summands above the initial state, which was $n_{\text {sum }}=4000$ for $n_{i} \in\{2,3\}$ and $n_{\text {sum }}=200$ for $n_{i}>3$.

\subsection{The two-photon rates for the ns and nd-states}

For future computations and more complete considerations of the higher order correction to the lifetime of the $n \mathrm{~s}$ and $n \mathrm{~d}$-states, here we now give the results for the total contribution of the nonresonant term to the two-photon decay rate. This contribution does not depend on the treatment of the poles in the cascade and interference terms. However, these values should have no direct relation to the total two-photon correction of the lifetime, but are mainly meant for cross-checks.

\subsubsection{The non-resonant contribution}

In Table 1 we summarize the values for the non-resonant contribution to the two-photon rates for the $n \mathrm{~s}$ and $n$ d-states up to $n_{i}=20$. The dependence of the non-resonant contribution to the total two-photon decay rate on $n_{i}$ is presented in Fig. 4. For large $n_{i}$ in both cases, the rates scale roughly linear, increasing towards larger $n_{i}$. The slope is slightly steeper for the s-states. We find that for $n_{i} \gtrsim 20$ one can use

$A_{n_{i} \mathrm{~s} \rightarrow 1 \mathrm{~s}}^{\mathrm{nr}} \approx-15.857 \mathrm{~s}^{-1}+3.930 \mathrm{~s}^{-1} n_{i}$

$A_{n_{i} \mathrm{dr} \rightarrow 1 \mathrm{~s}}^{\mathrm{nr}} \approx 10.432 \mathrm{~s}^{-1}+1.636 \mathrm{~s}^{-1} n_{i}$

within percent accuracy up to $n_{i} \sim 40$. Explicitly computing the values for $n_{i}=50\left(n_{\text {sum }}=200\right)$, we find $A_{50 \mathrm{~s} \rightarrow 1 \mathrm{~s}}^{\mathrm{nr}}=181.74 \mathrm{~s}^{-1}$ and $A_{50 \mathrm{~d} \rightarrow 1 \mathrm{~s}}^{\mathrm{nr}}=92.19 \mathrm{~s}^{-1}$, using our full numerical treatment, and $180.64 \mathrm{~s}^{-1}$ and $92.23 \mathrm{~s}^{-1}$ with the approximations (15). We did not check up to which value of $n_{i}$ the formulae (15) are applicable. Also, one should bear in mind that, above some value of $n_{i} \gg 1$, the usual dipole approximation for the transition matrix elements breaks down (Dubrovich \& Grachev 2005; Hirata \& Switzer 2007) and other methods should be used.

The linear scaling of the non-resonant contribution to the two-photon decay rate for $n_{i} \gg 1$ was expected (Dubrovich 1987; Dubrovich \& Grachev 2005), but here we have included all virtual intermediate states in the sum. However, one should keep in mind that, due to the interference term, it is difficult to interpret this contribution separately.

\section{Astrophysical application}

\subsection{Two-photon process in the single photon picture}

As described in the introduction, the standard procedure for treating the atomic transitions of electrons involving more than one photon is to break them down into independent, single-step, one-photon processes. This approximation should be especially good in the presence of many perturbing particles (free electrons and ions), such as in stellar atmospheres, which destroy the coherence of processes involving more than one transition. Here we now explain how the two-photon process can be formulated in the simplified $1+1$-single photon picture.

\subsubsection{Distribution of the high frequency photon}

As an example, we consider the decay of the $3 \mathrm{~s}$-level in vacuum. If there are no perturbing particles, two photons will be released and the emission profile (see Fig. 2) is described by the twophoton formulae discussed in the previous sections. In the $1+$ 1-single photon picture, with very high probability the electron after a short time $\left(\sim 1.6 \times 10^{-7} \mathrm{~s}\right)$ decays to the $2 \mathrm{p}$-state, emitting a photon close to the Balmer- $\alpha$ frequency. Then it independently releases a second photon, for which the frequency distribution, in the rest frame of the atom, is given by the natural line profile. Therefore the number of photons appearing per second in the frequency interval $v$ and $v+\mathrm{d} v$ in the vicinity of the Lyman$\alpha$ resonance due to the transition from the 3 s-state is given by ${ }^{10}$

$\phi_{2 \mathrm{p} \rightarrow 1 \mathrm{~s}}^{3 \mathrm{~s}, 1+1 \gamma}(v) \mathrm{d} v=\frac{A_{3 \mathrm{~s} \rightarrow 2 \mathrm{p}}^{1 \gamma}}{\pi} L\left(\frac{\Gamma_{2 \mathrm{p} \rightarrow 1 \mathrm{~s}}^{1 \gamma}}{4 \pi}, v-v_{\alpha}\right) \mathrm{d} v$

where $L(a, b)$ is defined in Eq. (12) and $v_{\alpha}$ is the Lyman- $\alpha$ central frequency. Integrating over $v$ yields $\int \phi_{2 \mathrm{p} \rightarrow 1 \mathrm{~s}}^{3 \mathrm{~s}, 1+1 \gamma}(v) \mathrm{d} v=A_{3 \mathrm{~s} \rightarrow 2 \mathrm{p}}^{1 \gamma}$, i.e. the total rate at which electrons are added to the $2 \mathrm{p}$-state

${ }^{10}$ The factor of $1 / \pi$ is required due to the normalization of $L(a, b)$. 
after the transition from the initial $3 \mathrm{~s}-$ level. Note that here we have assumed $\Gamma_{2 \mathrm{p}} \approx \Gamma_{2 \mathrm{p} \rightarrow 1 \mathrm{~s}}^{1 \gamma}$.

For Eq. (16) one assumes that there is no coherence or correlation between the first and second photon, and consequently the Lyman- $\alpha$ line-profile in the $1+1$-photon picture is a pure Lorentzian up to very large distances from the resonance. This is also equivalent to assuming that the transition from the $3 \mathrm{~s}$-state leads to a "natural" distribution of electrons within the 2 p-state (Mihalas 1978). One can also obtain this result using the interpretation of Weisskopf \& Wigner (1930) for the line width.

Looking at other initial s or d-states, the same argument as above can be carried out. In the more general case, one simply has to replace $A_{3 \mathrm{~s} \rightarrow 2 \mathrm{p}}^{1 \gamma}$ with the corresponding partial spontaneous decay rate $A_{n \mathrm{~s} / \mathrm{d} \rightarrow 2 \mathrm{p}}^{1 \gamma}$ to the $2 \mathrm{p}$-state. This shows that no matter what is the initial level, the shape of the $1+1$-emission profile in the vicinity of the Lyman- $\alpha$ resonance is always a Lorentzian. Within the $1+1$-single photon picture, the same is true for the other possible intermediate resonances (e.g Lyman- $\beta$, $\gamma$, etc.) in the two-photon cascades from high initial s or d-states. However, there in addition the partial width of the $2 p$-state due to the transition to the ground level appearing in Eq. (16) has to be replaced by the corresponding total (one-photon) width of the intermediate p-state. Also one has to take into account the branching ratio for transitions leading directly to the ground state.

With these comments one then can write

$\phi_{n \mathrm{p} \rightarrow 1 \mathrm{~s}}^{n_{i} \mathrm{~s} / \mathrm{d}, 1+1 \gamma}(v) \mathrm{d} v=\frac{A_{n_{i} \mathrm{~s} / \mathrm{d} \rightarrow n \mathrm{p}}^{1 \gamma}}{\pi} \frac{\Gamma_{n \mathrm{p} \rightarrow 1 \mathrm{~s}}^{1 \gamma}}{\Gamma_{n \mathrm{p}}^{1 \gamma}} L\left(\frac{\Gamma_{n \mathrm{p}}^{1 \gamma}}{4 \pi}, v-v_{n 1 \mathrm{~s}}\right) \mathrm{d} v$,

where $v_{n 1 \mathrm{~s}}$ is the central frequency of the corresponding Lymanseries transition.

\subsubsection{Distribution of the low-frequency photon}

In Sect. 5.1.1 we have focused on the high-frequency photons released in the two-photon cascade. If we now consider the lowfrequency photons, then the profiles of these will be given by

$\phi_{n_{i} \mathrm{~s} / \mathrm{d} \rightarrow n \mathrm{p}}^{1+1 \gamma}(v) \mathrm{d} v=\frac{A_{n_{i} \mathrm{~s} / \mathrm{d} \rightarrow n \mathrm{p}}^{1 \gamma}}{\pi} \frac{\Gamma_{n \mathrm{p} \rightarrow 1 \mathrm{~s}}^{1 \gamma}}{\Gamma_{n \mathrm{p}}^{1 \gamma}} L\left(\frac{\Gamma_{n \mathrm{p}}^{1 \gamma}}{4 \pi}, v-v_{n_{i} n \mathrm{p}}\right) \mathrm{d} v$,

where $v_{n_{i} n \mathrm{p}}=v_{n_{i} 1 \mathrm{~s}}-v_{n 1 \mathrm{~s}}$ is the transition frequency from the initial $n_{i} \mathrm{~s}$ or $n_{i} \mathrm{~d}$-state to the $n \mathrm{p}$-state.

Here one may ask why the width of the line is determined by the width of the intermediate $n \mathrm{p}$-state only and not by $\Gamma_{n \mathrm{p}}^{1 \gamma}+\Gamma_{n_{i} \mathrm{~s} / \mathrm{d}}^{1 \gamma}$ as usual. We simply wanted to be consistent with the approximate treatment of the cascade and interference terms in the full two-photon formulation (see Sect. 2.1.3), for which the width of the initial state was neglected. As mentioned above, physically this is motivated by the idea that, within the formulation of Weisskopf \& Wigner (1930), we consider one particular initial "energy sub-level" and do not specify the process that populated it. Therefore the final profile is independent of the shape of the distribution of energy-sub-levels around the mean energy of the initial state. One can also consider this as equivalent to neglecting any possible reshuffling of the electron by perturbing particles while it is in the initial state. Furthermore, in general $\Gamma_{n \mathrm{p}}^{1 \gamma}>\Gamma_{n_{i} \mathrm{~s} / \mathrm{d}}^{1 \gamma}$ such that $\Gamma_{n_{i} \mathrm{~s} / \mathrm{d}}^{1 \gamma}$ would not contribute much to the total width of the line. But most important, in our computations we do not approach the resonances so close that these differences would play any role.

\subsubsection{Total two-photon profile in the single photon picture}

With Eqs. (17) and (18) it is now possible to write the total distribution of photons emitted in the two-photon decay of an isolated hydrogen atom in some given initial s or d-level within the $1+1$-single photon formulation:

$\phi_{n_{i} \mathrm{~s} / \mathrm{d} \rightarrow 1 \mathrm{~s}}^{1+1 \gamma}(v)=\sum_{n=2}^{n_{i}-1}\left[\phi_{n_{i} \mathrm{~s} / \mathrm{d} \rightarrow n \mathrm{p}}^{1+1 \gamma}(v)+\phi_{n \mathrm{p} \rightarrow 1 \mathrm{~s}}^{n_{i} \mathrm{~s} / \mathrm{d}, 1+1 \gamma}(v)\right]$.

Integrating $\phi_{n_{i} \mathrm{~s} / \mathrm{d} \rightarrow 1 \mathrm{~s}}^{1+1 \gamma}(v)$ over all possible frequencies yields $\frac{1}{2} \int \phi_{n_{i} \mathrm{~s} / \mathrm{d} \rightarrow 1 \mathrm{~s}}^{1+1 \gamma}(v) \mathrm{d} v \equiv \sum_{n=2}^{n_{i}-1} A_{n_{i} \mathrm{~s} / \mathrm{d} \rightarrow n \mathrm{p}}^{1 \gamma} \frac{\Gamma_{n p \rightarrow 1 \mathrm{~s}}^{1 \gamma}}{\Gamma_{n \mathrm{p}}^{1 \gamma}}$, which is the total contribution to the width of the initial level due to spontaneous transitions that lead to the ground state and involve exactly two photons (cf. Eq. (9)).

What are the main differences of the 1+1-photon profile with respect to the full two-photon profile, as defined by Eq. (7)?

(i) There is no non-resonant contribution, resulting from virtual transitions via intermediate states with energies higher than or equal to the initial state.

(ii) As a consequence of (i) there is no resonance/nonresonance interference term.

(iii) In contrast to Eq. (14a), there is no interference among the resonances.

(iv) As a consequence of (ii) and (iii) each resonance has the shape of a Lorentzian up to very large distances from their line centers.

(v) Usually one does not restrict the range of integration to the interval $0 \leq v \leq v_{n 1 \mathrm{~s}}$.

It is also important that interpreting each resonance appearing in $\phi_{n_{i} \mathrm{~s} / \mathrm{d} \rightarrow 1 \mathrm{~s}}^{1+1 \gamma}(v)$ separately, it is possible to uniquely define the rates at which electrons flow in and out of a particular intermediate p-state. In astrophysical computations this is the usual procedure for solving the radiative transfer problem for each transition separately. Within the full two-photon formulation this is only possible very close to the centers of the resonances (where the contribution from the other terms is negligible), but in the wings, photons from different intermediate transitions contribute nontrivially and make this separation difficult. Also this overlap of the resonances is taken into account in Eq. (19), but it is usually neglected in astrophysical computations.

As an example, we illustrate the differences in the twophoton emission profiles for the initial $4 \mathrm{~s}$ and $4 \mathrm{~d}$ states in Fig. 5. One can see that in the distant wings of all the resonances the differences of the profiles are rather big. This is mainly due to the non-resonant term and its interference with the cascade contribution, but also the resonance/resonance interference plays some role. Below we now focus our analysis on the deviations of the two-photon profile from the pure Lorentzian close to the Lyman- $\alpha$ resonance. These differences are the main reason for the corrections to the hydrogen recombination history.

\subsection{Two-photon profiles close to the Lyman- $\alpha$ resonance}

In low-density plasmas like the expanding Universe during cosmological hydrogen recombination, hardly any perturbing particle is within the Weisskopf-radius (Weisskopf 1932; Sobelman et al. 1995), so that the coherence in two-photon and possibly multi-photon transitions is maintained at least for the lower shells. In astrophysical computations the frequency distribution of photons released in the Lyman- $\alpha$ transition due to electrons 

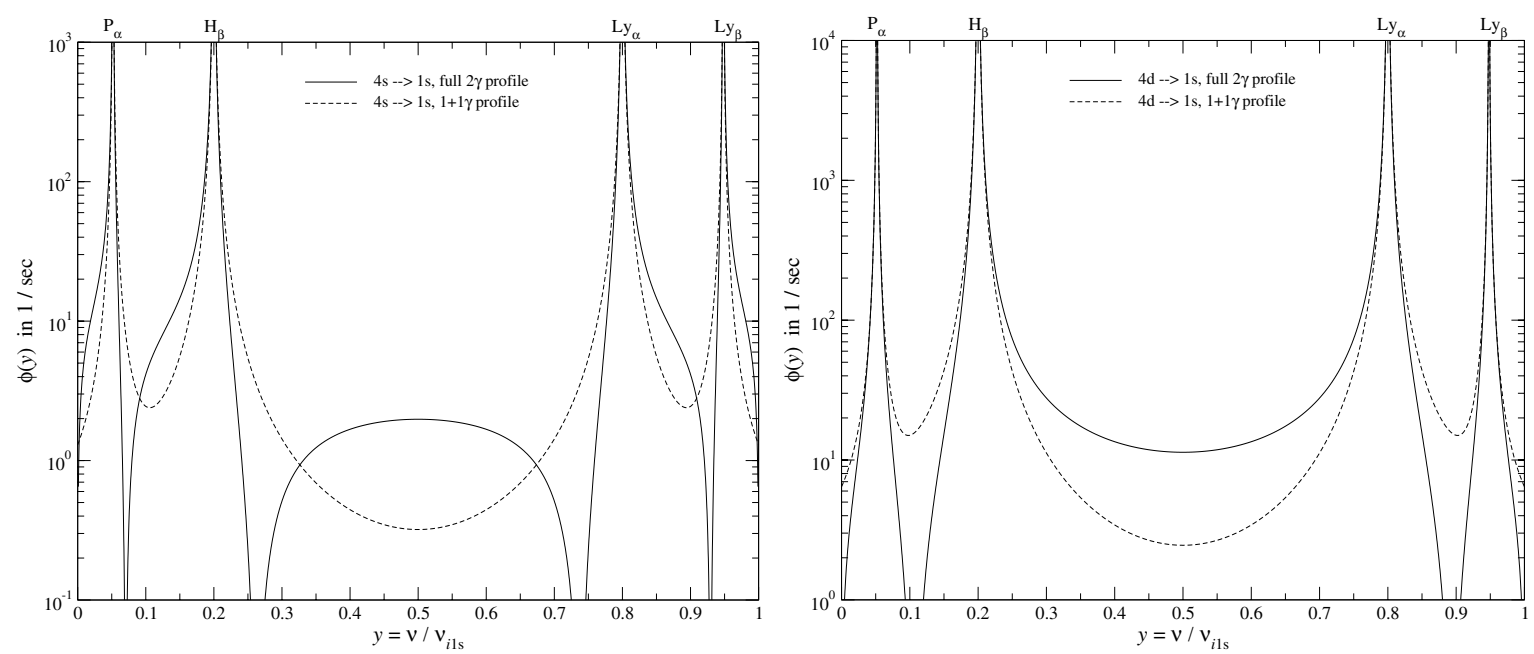

Fig. 5. Comparison of the two-photon emission profiles for the $4 \mathrm{~s}$ and $4 \mathrm{~d}$ states. We show, $\phi_{4 \mathrm{~s} / \mathrm{d} \rightarrow 1 \mathrm{~s}}^{2 \gamma}$, following from the full $2 \gamma$ treatment according to Eq. (7), and, $\phi_{4 \mathrm{~s} / \mathrm{d} \rightarrow 1 \mathrm{~s}}^{1+1 \gamma}$, using the $1+1$-single photon description, as given by Eq. (19). The first 200 terms above $n=4$ were included in the sum for $\phi_{4 \mathrm{~s} / \mathrm{d} \rightarrow 1 \mathrm{~s}}^{2 \gamma}$.

reaching the $2 \mathrm{p}$-state from higher levels is usually described by a pure Lorentzian. Within the interpretation of Weisskopf \& Wigner (1930), this means that the electron is completely reshuffled among all the possible $2 p$ energy sub-levels.

In calculations of the cosmological hydrogen recombination problem, we are now interested in the deviations of the full two-photon profile from the normal Lorentzian shape. Here one should mention that in general the deviations of the $2 \gamma$-emission profile, using the full two-photon treatment as described in Sect. 2, from the one in the $1+1$-single photon description (Eq. (19)) should also be considered. However, in the red wing of the Lyman- $\alpha$ resonance, one can write

$$
\begin{aligned}
\phi_{n_{i} \mathrm{~s} / \mathrm{d} \rightarrow 1 \mathrm{~s}}^{1+1 \gamma} & \approx \sum_{n=2}^{n_{i}-1}\left[\frac{A_{n_{i} \mathrm{~s} / \mathrm{d} \rightarrow n \mathrm{p}}^{1 \gamma}}{4 \pi^{2}} \frac{\Gamma_{n \mathrm{p} \rightarrow 1 \mathrm{~s}}^{1 \gamma}}{\left[v-v_{n_{i} n \mathrm{p}}\right]^{2}}+\frac{A_{n_{i} \mathrm{~s} / \mathrm{d} \rightarrow n \mathrm{p}}^{1 \gamma}}{4 \pi^{2}} \frac{\Gamma_{n \mathrm{p} \rightarrow 1 \mathrm{~s}}^{1 \gamma}}{\left[v-v_{n 1 \mathrm{~s}}\right]^{2}}\right] \\
& \approx \frac{A_{n_{i} \mathrm{~s} / \mathrm{d} \rightarrow 2 \mathrm{p}}^{1 \gamma} \Gamma_{2 \mathrm{p} \rightarrow 1 \mathrm{~s}}^{1 \gamma}}{4 \pi^{2}\left[v-v_{2 \mathrm{p} 1 \mathrm{~s}}\right]^{2}}
\end{aligned}
$$

where the last step is possible, since the distant wings of all the other resonances do not contribute significantly when one is close enough to the center of the Lyman- $\alpha$ transition. The more one approaches the Lyman- $\alpha$ resonance, the better this approximation becomes. For the estimates carried out below, this approximation is satisfactory.

To understand the deviations of the two-photon emission profiles close to the Lyman- $\alpha$ resonance, we now directly compare $\phi_{n \mathrm{~s} / \mathrm{d} \rightarrow 1 \mathrm{~s}}^{2 \gamma}$ according to Eq. (7) with $\phi_{2 \mathrm{p} \rightarrow 1 \mathrm{~s}}^{n_{i} \mathrm{~s} / \mathrm{d}, 1+1 \gamma}$ as given by Eq. (17). For convenience we choose $y_{\alpha}=v / v_{\alpha}$ as the common frequency variable. Then the full two-photon profile in this new coordinate is given by $\phi_{\alpha}^{2 \gamma}\left(y_{\alpha}\right)=v_{\alpha} \phi_{n \mathrm{~s} / \mathrm{d} \rightarrow 1 \mathrm{~s}}^{2 \gamma}(y) / v_{i 1 \mathrm{~s}}$. The axis of symmetry is then at $y_{\alpha}=\frac{2}{3}\left[n^{2}-1\right] n^{-2}$ instead of $y=1 / 2$. Since in the vicinity of any particular resonance all the two-photon profiles scale like $\Gamma_{n \mathrm{~s} / \mathrm{d} \rightarrow n^{\prime} \mathrm{p}}$, focusing on the Lyman- $\alpha$ transition, we also re-normalized by $\Gamma_{n \mathrm{~s} / \mathrm{d} \rightarrow 2 \mathrm{p} \text {. }}$.

In Fig. 6 we give the normalized $1+1$-two-photon profile in the vicinity of the Lyman- $\alpha$ transition in comparison with the renormalized two-photon-profiles for several initial s and d-states.
One can see that at large distances the two-photon profiles in the full $2 \gamma$-treatment deviate a lot from the Lorentzian shape. For both the initial s and d-states, the very distant red wing is several times above the Lorentzian. Within the frequency range $0.9 \lesssim y_{\alpha} \lesssim 1.1$ for the s-states, the red wing lies below, the blue wing above the Lorentzian, whereas the opposite is true for the d-states. In particular for the d-states, the red wing is always above the Lorentzian, and unlike the s-states in the considered frequency range there is no additional zero below the Lyman- $\alpha$ resonance. In Fig. 6 one can also see that for the chosen set of coordinates, the variation in the profiles is rather small in the case of initial s-states, and the modifications become negligible even for initial d-states above $n \sim 10$.

In Fig. 7 we show the relative difference of the curves given in Fig. 6 with respect to the Lorentzian of the Lyman- $\alpha$ resonance. The wing redward of the Lyman- $\alpha$ frequency lies below the Lorentzian for initial s-states, exceeding the level of $\sim 10 \%$ at more than $\sim 1.6 \times 10^{4}$ natural width from the center. For initial $\mathrm{d}$-states, in all shown cases the wing redward of the Lyman- $\alpha$ frequency lies above the Lorentzian. The relative correction to the Lorentzian scales roughly linearly with $\Delta v=v-v_{\alpha}$ in this regime. Therefore the net change in the rate of photon production in the red wing of the Lyman- $\alpha$ transition at frequencies in the range $v_{1} \lesssim v \lesssim v_{2}$ depends logarithmically on the ratio of $v_{1}$ and $v_{2}: \Delta N_{\gamma} \propto \int_{v_{1}}^{v_{2}} \frac{1}{\left[v-v_{\alpha}\right]^{2}} \times \Delta v \mathrm{~d} v \propto \log \left[v_{2} / v_{1}\right]$. Here we used the wing approximation of the Lorentzian $L \propto 1 /\left[v-v_{\alpha}\right]^{2}$. This estimate shows that the value of the effective two-photon decay rate does not depend very strongly on $v_{2}$ (see Sect. 5.3).

\subsection{Cosmological hydrogen recombination}

\subsubsection{Escape of photons in the red wing of the Lyman- $\alpha$ resonance}

In the context of cosmological hydrogen recombination, the escape of photons in the red wing of the Lyman- $\alpha$ resonance, which is one of the major channels to reach the ground state of hydrogen, plays a key role in controlling the dynamics of recombination (Varshalovich \& Syunyaev 1968; Grachev \& Dubrovich 1991; Rybicki \& dell' Antonio 1994; Chluba \& Sunyaev 2007a). At large distances, say at frequencies below $v_{\mathrm{c}}$ redward of the 

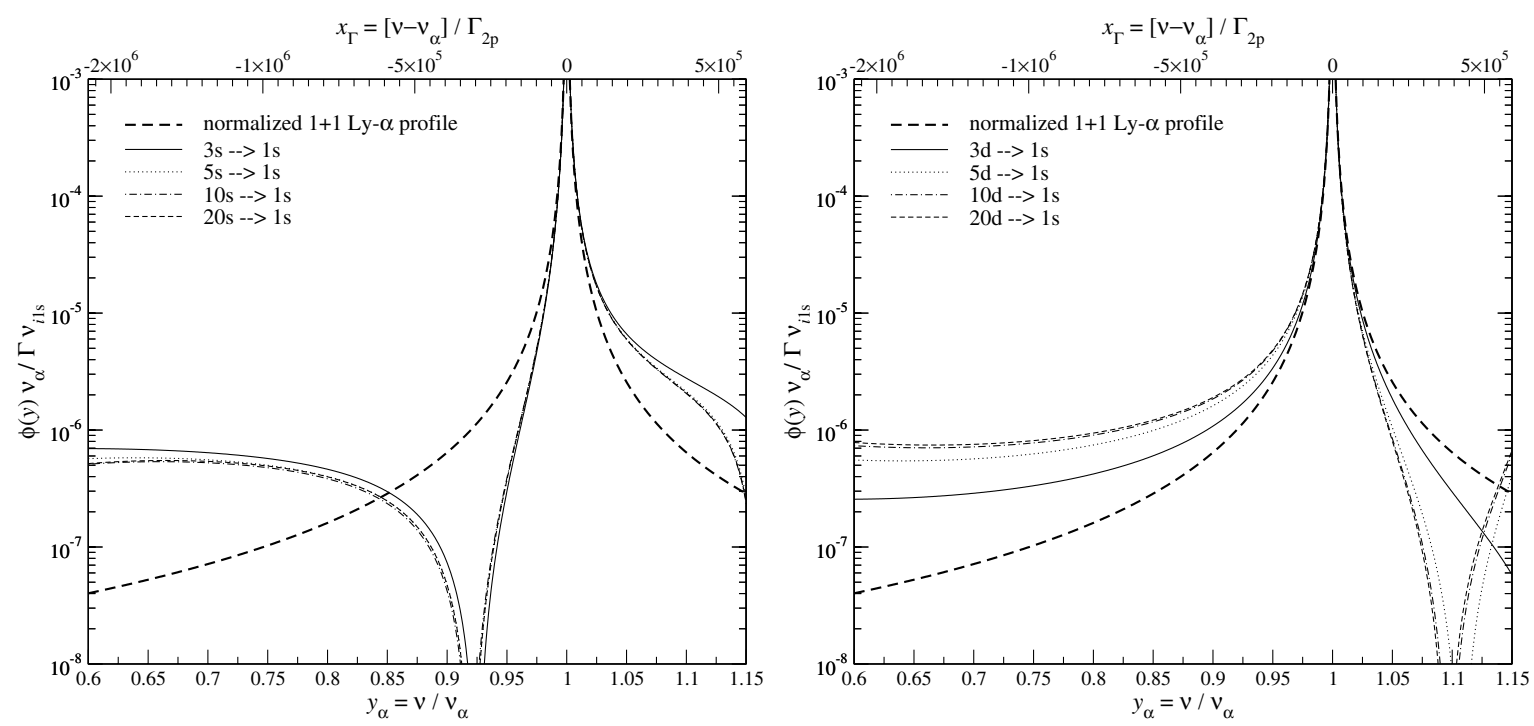

Fig. 6. Normalized 1+1-two-photon profile $\phi_{2 p \rightarrow 1 \mathrm{~s}}^{n \mathrm{~s} / \mathrm{d}, 1 \gamma}$, see Eq. (17), close to the Lyman- $\alpha$ frequency in comparison with the full two-photon-profiles, $\phi_{n s / d \rightarrow 1 s}^{2 \gamma}$ according to Eq. (7), for several initial s and d-states. We divided the two-photon spectra by their partial one-photon transition rate to the $2 \mathrm{p}$-state $\left(\Gamma_{n s / \mathrm{d} \rightarrow 2 \mathrm{p}}\right)$ and transformed to the variable $y_{\alpha}=v / v_{\alpha}$. For the given curves we included the first 500 terms above $n_{i}$. The dashed curve corresponds to the usual Lorentzian profile of the Lyman- $\alpha$ transition.
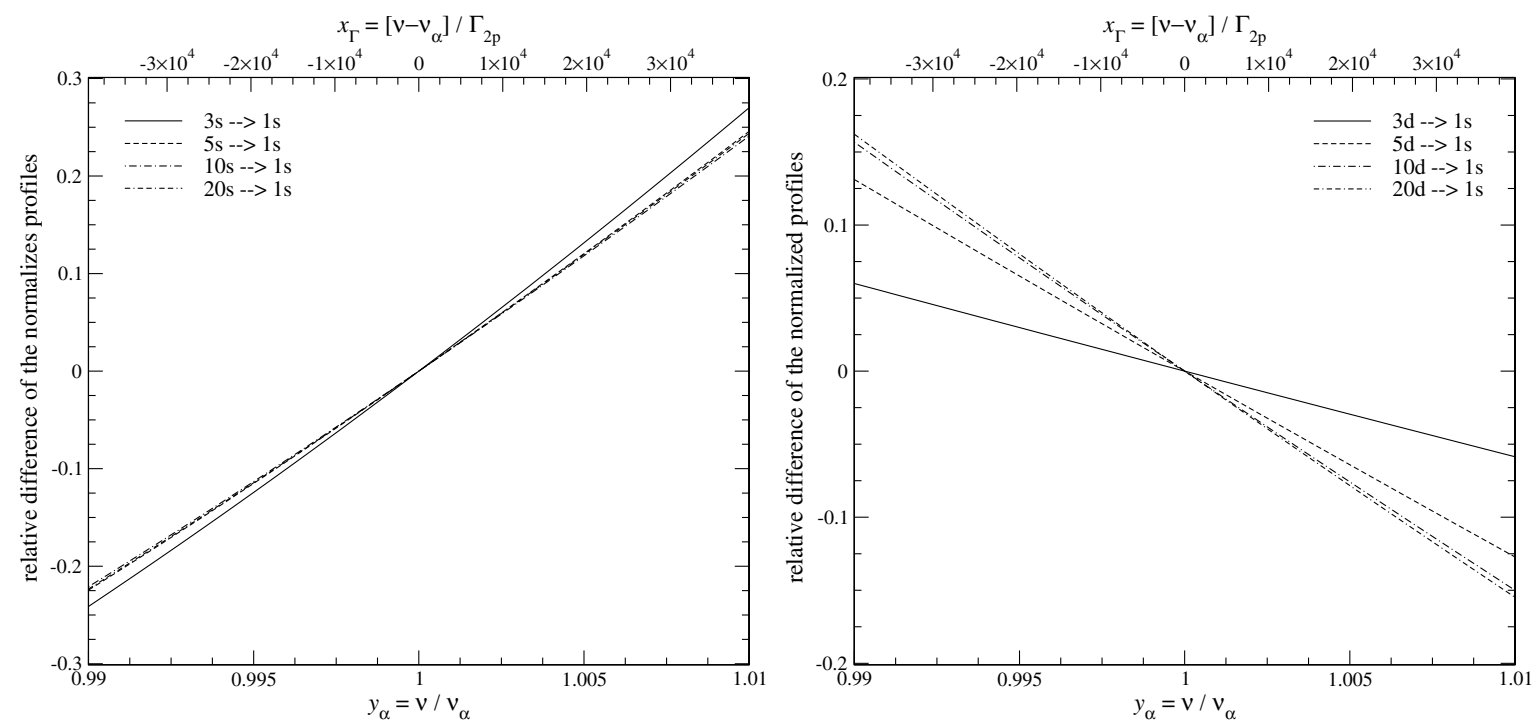

Fig. 7. Relative difference, $\left[v_{\alpha} \phi_{n \mathrm{~s} / \mathrm{d} \rightarrow 1 \mathrm{~s}}^{2 \gamma}\left(y_{\alpha}\right) / v_{n 1 \mathrm{~s}}-\phi_{2 \mathrm{p} \rightarrow 1 \mathrm{~s}}^{n s / \mathrm{d} 1+1 \gamma}\left(y_{\alpha}\right)\right] / \phi_{2 \mathrm{p} \rightarrow 1 \mathrm{~s}}^{n \mathrm{~s} / \mathrm{l} / \mathrm{l} \gamma}\left(y_{\alpha}\right)$, of the curves given in Fig. 6 with respect to normalized $1+1$-twophoton profile. Very close to $y_{\alpha} \sim 1$, the curves should be considered as extrapolated estimates.

Lyman- $\alpha$ central frequency, $v_{\alpha}$, the probability of absorbing a photon to the continuum, thereby creating a free electron, becomes very low. Photons released below $v_{\mathrm{c}}$ directly escape further interaction with the neutral hydrogen atoms and lead to the settling of an electron in the 1 s-state. On the other hand, all photons emitted at frequencies $v \gtrsim v_{\mathrm{c}}$ will have a very high probability of being absorbed in the continuum or undergoing transitions to higher levels, possibly after many interactions with neutral hydrogen atoms or when redshifting into the domain of the Lyman- $\alpha$ resonance from frequencies $v>v_{\alpha}$.

Determining the exact value of $v_{\mathrm{c}}$ during the epoch of cosmological hydrogen recombination requires a full treatment of the radiative transfer in the Lyman- $\alpha$ resonance. Our computations show (Chluba \& Sunyaev 2007a) that $v_{\mathrm{c}}$ depends on redshift and should typically lie within 100 to 1000 Doppler width below the Lyman- $\alpha$ frequency. At redshift $z$, one Doppler width corresponds to

$$
\begin{aligned}
\Delta v_{\mathrm{D}} & \approx 58.0\left[\frac{(1+z)}{1100}\right]^{1 / 2} \mathrm{GHz} \approx 2.35 \times 10^{-5}\left[\frac{(1+z)}{1100}\right]^{1 / 2} v_{\alpha} \\
& \approx 92.5\left[\frac{(1+z)}{1100}\right]^{1 / 2} \Gamma_{2 \mathrm{p} \rightarrow 1 \mathrm{~s}}^{1 \gamma} ;
\end{aligned}
$$

hence $\left|v_{\mathrm{c}}-v_{\alpha}\right| / v_{\alpha} \sim 10^{-3}-10^{-2}$, or in terms of the $2 \mathrm{p}$-line width $\left|v_{\mathrm{c}}-v_{\alpha}\right| / \Gamma_{2 \mathrm{p} \rightarrow 1 \mathrm{~s}}^{1 \gamma} \sim 10^{4}-10^{5}$.

In computations of the hydrogen recombination history, it is therefore important to know how many photons reach the very distant red wing of the Lyman- $\alpha$ resonance directly. If we want to estimate this effect, we need to compute the difference in the number of photons, that are directly escaping in the distant wing 
by comparing the emission profiles in the full treatment of twophoton processes with the one in $1+1$-single photon picture. This will show the relevance of this process.

If we consider those photons emitted in the red wing of the Lyman- $\alpha$ resonance because of two-photon transitions from upper s or d-states, then when introducing the dimensionless frequency variable $x_{\Gamma}(v)=\left[v-v_{\alpha}\right] / \Gamma_{2 p}$, the results discussed in Sect. 5.2 suggest the following:

(i) Because of two-photon processes, more photons will escape in the very distant red wing of the Lyman- $\alpha$ resonance (below $x_{\Gamma} \sim-5 \times 10^{5}$ ) than in the $1+1$-single photon treatment for the direct cascade emission.

(ii) For initial s-states, fewer photons are emitted in the range $-5 \times 10^{5} \lesssim x_{\Gamma} \lesssim-10^{4}$ than in the $1+1$-single photon picture.

(iii) For initial d-states, more photons are emitted in the range $-5 \times 10^{5} \lesssim x_{\Gamma} \lesssim-10^{4}$ than in the $1+1$-single photon picture.

Because of (i) and (iii) hydrogen recombination should occur slightly faster, while (ii) may make it a bit slower. Since the statistical weight of d-states is 5 times higher than for s-levels, one expects that recombination will in total be slightly faster than in the standard treatment because of two-photon processes.

In addition to the direct escape of photons in the distant red wing of the Lyman- $\alpha$ transition, also significant differences close to the line center arise (see Fig. 7). Understanding how these changes affect the effective escape of photon from the line center requires a more rigorous treatment of the radiative transfer problem in the line. Also the feedback of photons emitted in the blue wing of the Lyman- $\alpha$ transition and in particular those coming from the other Lyman-series transitions, should be slightly modified when taking the full two-photon process into account. Both aspects are beyond the scope of this paper and will be addressed in a future work.

We can now estimate the effect of the changes in the effective escape of photons in the distant red wing of the Lyman- $\alpha$ transition. For this only the photons between the innermost resonances in the two-photon emission spectrum are contributing (e.g. photons between the Balmer- $\gamma$ and Lyman- $\alpha$ transition for the $5 \mathrm{~s}$ and 5d-two-photon decay, see Fig. 3). This is because we only want to count photons up to $v \leq v_{\mathrm{c}}$ and correspondingly $v^{\prime} \geq v_{n 1 \mathrm{~s}}-v_{\mathrm{c}}$. Because of the symmetry of the full two-photon profile, it is therefore sufficient to integrate $\phi_{n \mathrm{~s} / \mathrm{d} \rightarrow 1 \mathrm{~s}}^{2 \gamma}(y)$ from $y=1 / 2$ up to $y_{\mathrm{c}}=v_{\mathrm{c}} / v_{i 1 \mathrm{~s}}$ :

$A_{n \mathrm{~s} / \mathrm{d} \rightarrow 1 \mathrm{~s}}^{2 \gamma}\left(\nu_{\mathrm{c}}\right)=\frac{1}{2} \int_{1-y_{\mathrm{c}}}^{y_{\mathrm{c}}} \phi_{n \mathrm{~s} / \mathrm{d} \rightarrow 1 \mathrm{~s}}^{2 \gamma}(y) \mathrm{d} y \equiv \int_{1 / 2}^{y_{\mathrm{c}}} \phi_{n \mathrm{~s} / \mathrm{d} \rightarrow 1 \mathrm{~s}}^{2 \gamma}(y) \mathrm{d} y$.

This integral yields the total number of photons that directly escape per second in the distant red wing of the Lyman- $\alpha$ line. It should be compared with the value computed using the standard $1+1$-single photon profile. Since we only consider cases very far in the red wing of the Lyman- $\alpha$ transition, the integral over the Lorentzian resulting in the $1+1$ approach can be written as

$A_{2 \mathrm{p} \rightarrow 1 \mathrm{~s}}^{n \mathrm{~s} / \mathrm{d}, 1+1 \gamma}\left(v_{\mathrm{c}}\right)=\int_{0}^{v_{\mathrm{c}}} \phi_{2 \mathrm{p} \rightarrow 1 \mathrm{~s}}^{n \mathrm{~s} / \mathrm{d}, 1+1 \gamma}(v) \mathrm{d} v \approx \frac{A_{n \mathrm{~s} / \mathrm{d} \rightarrow 2 \mathrm{p}}}{4 \pi^{2}} \frac{1+x_{\Gamma, \mathrm{c}} \epsilon}{-x_{\Gamma, \mathrm{c}}}$

where we introduced $\epsilon=\Gamma_{2 \mathrm{p} \rightarrow 1 \mathrm{~s}} / v_{\alpha} \approx 2.540 \times 10^{-7}$ and used the variable $x_{\Gamma}(v)=\left[v-v_{\alpha}\right] / \Gamma_{2 \mathrm{p}}$. The effective difference in the photon production rate, or equivalently the photon escape rate in the distant wings, is then given by

$\Delta A_{n \mathrm{~s} / \mathrm{d} \rightarrow 1 \mathrm{~s}}\left(v_{\mathrm{c}}\right)=A_{n \mathrm{~s} / \mathrm{d} \rightarrow 1 \mathrm{~s}}^{2 \gamma}\left(v_{\mathrm{c}}\right)-A_{2 \mathrm{p} \rightarrow 1 \mathrm{~s}}^{n \mathrm{~s} / 1+1 \gamma}\left(v_{\mathrm{c}}\right)$ for a fixed frequency $v_{\mathrm{c}}$. Although in general $v_{\mathrm{c}}$ is a function of time, below we assume that it is constant. A more rigorous treatment will be presented in some future work.

\subsubsection{Approximate inclusion into the multi-level code}

In our formulation, $\Delta A_{n \mathrm{~s} / \mathrm{d} \rightarrow 1 \mathrm{~s}}\left(v_{\mathrm{c}}\right)$ plays the role of the pure twophoton rate coefficients used in Dubrovich \& Grachev (2005) and Wong \& Scott (2007). If we want to estimate the possible impact of our results on the hydrogen recombination history, we have to take the additional net escape of photons into account. This can be accomplished by adding

$\Delta R_{n \mathrm{~s} / \mathrm{d} \rightarrow 1 \mathrm{~s}}^{2 \gamma} \approx \Delta A_{n \mathrm{~s} / \mathrm{d} \rightarrow 1 \mathrm{~s}}\left(v_{\mathrm{c}}\right)\left[N_{n \mathrm{~s} / \mathrm{d}}-\frac{g_{n \mathrm{~s} / \mathrm{d}}}{g_{1 \mathrm{~s}}} N_{1 \mathrm{~s}} \mathrm{e}^{-h v_{n 1 \mathrm{~s}} / k T_{\gamma}}\right]$

to the rate equation of the 1 s-state and subtracting it from the corresponding rate equation of the $n \mathrm{~s}$ and $n \mathrm{~d}$-levels. Here $N_{1 \mathrm{~s}}$, $N_{n \mathrm{~s}}, N_{n \mathrm{~d}}$ are the number density of hydrogen atom in the $1 \mathrm{~s}$, $n \mathrm{~s}$, and $n \mathrm{~d}$-states, respectively. Furthermore, $T_{\gamma}=T_{0}(1+z)$, is the temperature of the ambient blackbody radiation field, with $T_{0}=2.725 \mathrm{~K}$ (Fixsen \& Mather 2002). The factors $g_{n \mathrm{~s}} \equiv g_{1 \mathrm{~s}}$ and $g_{n \mathrm{~d}} \equiv 5 g_{1 \mathrm{~s}}$ are due to the statistical weights of the s and d-states. In (25) we have neglected any possible deviation in the radiation field from a blackbody and also omitted stimulated two-photon emission. Both processes should only lead to higher order corrections. Moreover we have added an inverse term, assuming detailed balance. This term is not important during the main epoch of hydrogen recombination $(z \lesssim 1600)$ and was only included to maintain full thermodynamic equilibrium at high redshifts. A self-consistent derivation is beyond the scope of this paper.

\subsubsection{Results for the photon production in the distant wings}

In Fig. 8 we give the rate of photon production at frequencies below $x_{\Gamma}$ within the full two-photon treatment, i.e. according to Eq. (22), for several initial s and d-states. For the d-states the photon production is $\sim 10$ times faster than for the corresponding s-state. In the case of initial s-states, the plateau of $A_{n \mathrm{~s} \rightarrow 1 \mathrm{~s}}^{2 \gamma}\left(v_{\mathrm{c}}\right)$ close to $x_{\Gamma} \sim-4 \times 10^{5}$ is caused by the zero in the central region of the two-photon emission spectra (e.g. see Fig. 6). As mentioned in Sect. 3, this zero is absent in the two-photon spectra of initial d-states, and consequently no such plateau appears for $A_{n d \rightarrow 1 s}^{2 \gamma}\left(v_{\mathrm{c}}\right)$. In both cases the rate of photon production decreases when increasing $n$. Looking at Fig. 4, just from the non-resonant term one would expect the opposite behavior. However, due to destructive interference this does not happen.

In Fig. 9 the net change in the rate of photon production at frequencies below $x_{\Gamma}$ is shown. The photon production due to the two-photon decay of initial s-states, at relevant distances from the Lyman- $\alpha$ center $\left(x_{\Gamma}>-10^{5}\right)$, is actually slower than in the $1+1$-single photon picture. This suggests that due to the full treatment of the two-photon process for the s-states alone, cosmological hydrogen recombination is expected to be slower than in the standard computations. This contrasts to the work of Wong \& Scott (2007), where both the s and d-state two-photon process leads to an increase in the rate of recombination.

On the other hand, for the d-states the effective photon escape rate is higher than in the $1+1$-single photon picture, hence one expects an increase in the rate of recombination. Since the statistical weights of the d-states are 5 times larger than the s-states, and also the effective increase in the wing photon production rate is roughly additional 5 times higher (cf. Fig. 9), one still expects that, even when including the combined effect of 

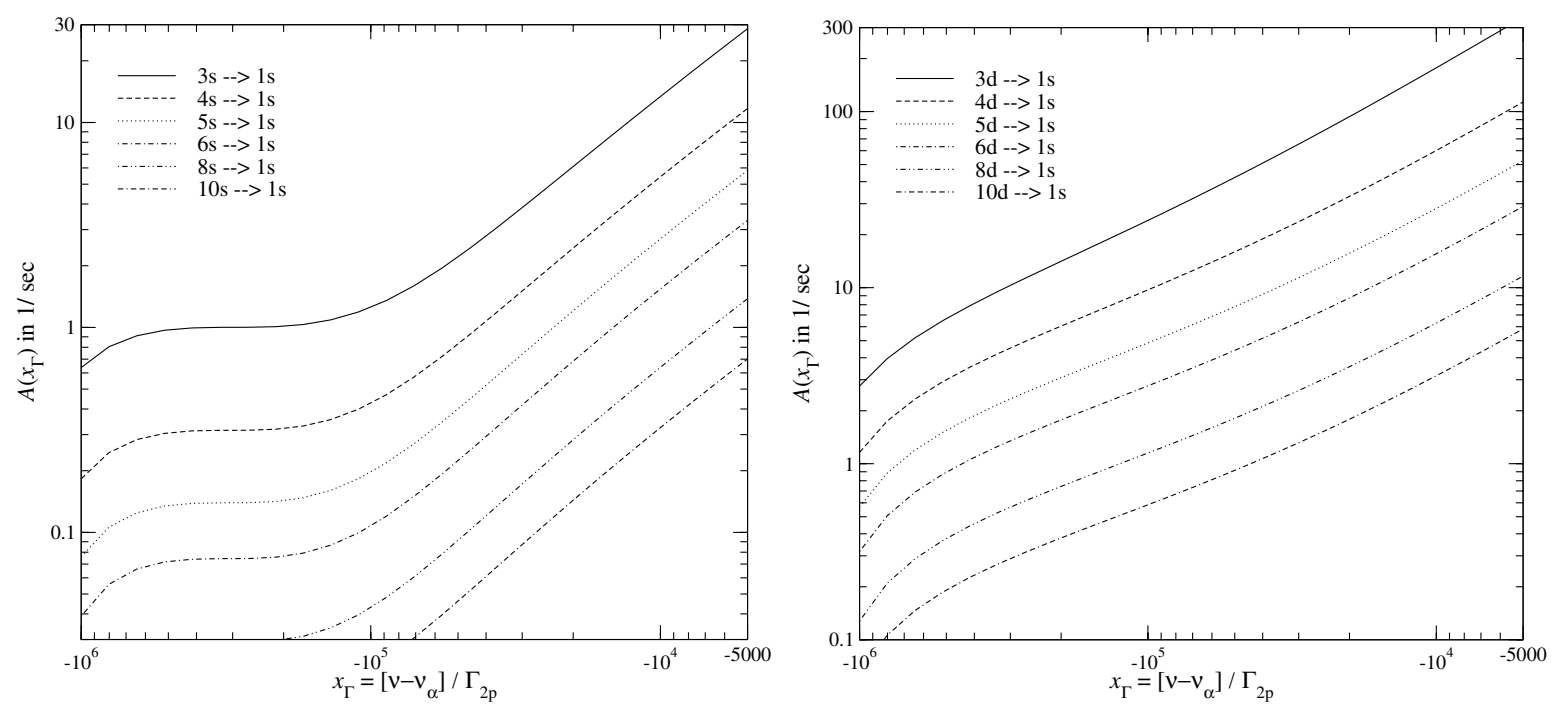

Fig. 8. Rate of photon production at frequencies below $x_{\Gamma}$ according to Eq. (22) for several initial $s$ and d-states. To convert to the variable $\left[v-v_{\alpha}\right] / v_{\alpha}$, one should multiply $x_{\Gamma}$ by $\sim 2.54 \times 10^{-7}$.
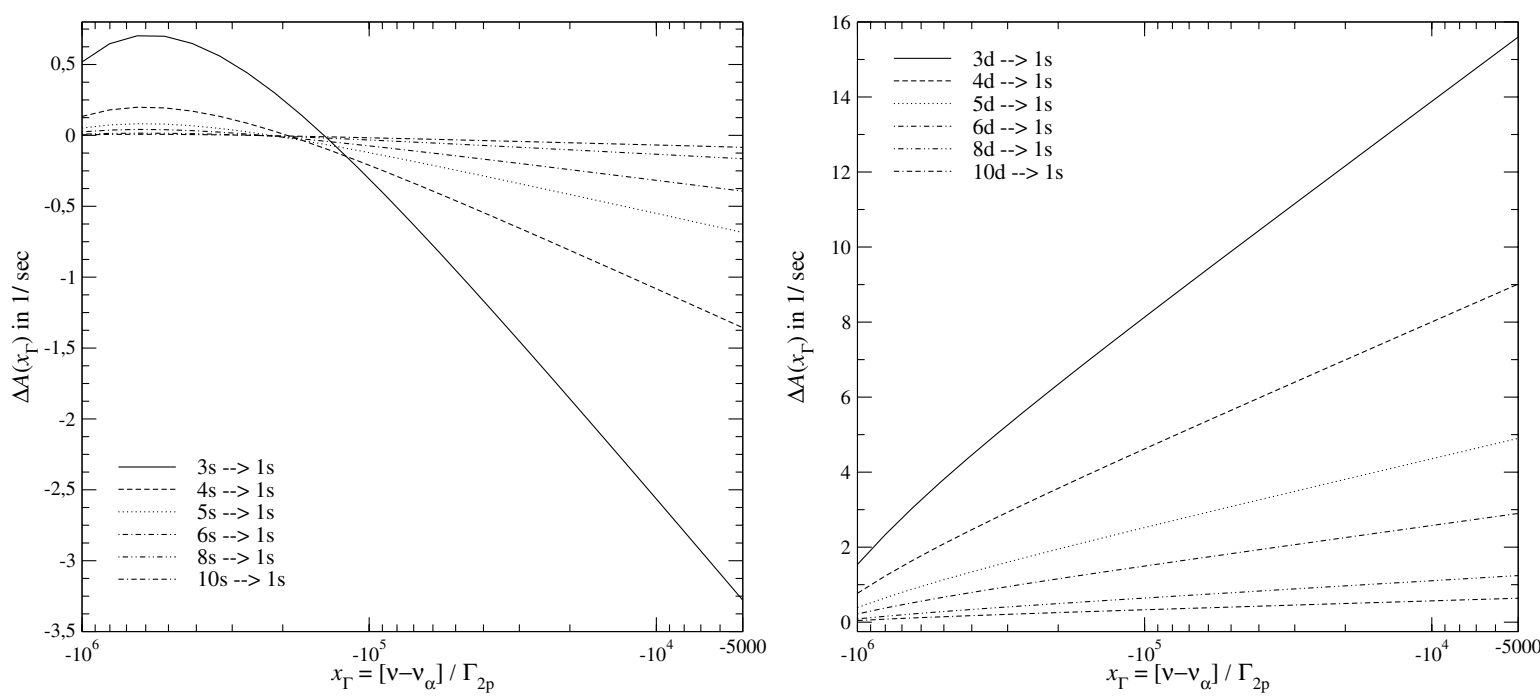

Fig. 9. Effective change in the rate of photon production (real profile minus Lorentzian) at frequencies below $x_{\Gamma}$, according to Eq. (24). To convert to the variable $\left[v-v_{\alpha}\right] / v_{\alpha}$, one should multiply $x_{\Gamma}$ by $\sim 2.54 \times 10^{-7}$.

the $\mathrm{s}$ and d-state, two-photon process, cosmological hydrogen recombination in total will proceed faster than in the standard treatment.

We would like to mention that using the analytic approximations given in Appendix B for the non-resonant term in connection with the formulae in Sect. 2.1.3 we were able to reproduce the rates presented in this section.

\subsubsection{Comparing with earlier works}

In the works of Dubrovich \& Grachev (2005) and Wong \& Scott (2007), only the combined effect of the two-photon process for the $n$ s and $n$ d-states on the hydrogen recombination history was discussed. To compare our results for the effective photon production rates with their values, we also write the combined effective decay rate

$6 \Delta A_{n \mathrm{~s}+n \mathrm{~d} \rightarrow 1 \mathrm{~s}}\left(v_{\mathrm{c}}\right)=\Delta A_{n \mathrm{~s} \rightarrow 1 \mathrm{~s}}\left(v_{\mathrm{c}}\right)+5 \Delta A_{n \mathrm{~d} \rightarrow 1 \mathrm{~s}}\left(v_{\mathrm{c}}\right)$,

where we implicitly assumed that the $n$ s and $n$ d-states are in full statistical equilibrium with each other $\left(N_{n \mathrm{~d}}=5 N_{n \mathrm{~s}}\right)$. At the relevant redshift, the deviations from full statistical equilibrium are rather small (Rubiño-Martín et al. 2006; Chluba et al. 2007), so that this approximation is possible (see Sect. 5.3.5).

In Fig. 10 we present the results for $\Delta A_{n \mathrm{~s}+n \mathrm{~d} \rightarrow 1 \mathrm{~s}}\left(v_{\mathrm{c}}\right)$ for several shells. If we consider the effective rate for the $3 \mathrm{~s}$ and $3 \mathrm{~d}$-levels then, even for very conservative values of $v_{\mathrm{c}}$, say 1000 Doppler width or $\sim 10^{5}$ natural width below the Lyman$\alpha$ resonance, we obtain $\Delta A_{3 \mathrm{~s}+3 \mathrm{~d} \rightarrow 1 \mathrm{~s}} \sim 6.7 \mathrm{~s}^{-1}$, whereas from the formulae in Dubrovich \& Grachev (2005) and Wong \& Scott (2007) one can find $\Delta A_{3 \mathrm{~s}+3 \mathrm{~d} \rightarrow 1 \mathrm{~s}}^{\mathrm{DG}} \sim 22 \mathrm{~s}^{-1}$ and $\Delta A_{3 \mathrm{~s}+3 \mathrm{~d} \rightarrow 1 \mathrm{~s}}^{\mathrm{WS}} \sim$ $1.5 \mathrm{~s}^{-1}$, respectively. Our value is only $\sim 3.3$ times lower than the one of Dubrovich \& Grachev (2005) but $~ 4.5$ times higher than in Wong \& Scott (2007). We would argue that for the third shell even values up to $10 \mathrm{~s}^{-1}$ still are reasonable, in particular at very low $(z \lesssim 1000)$ and high $(z \gtrsim 1300)$ redshifts, where the probability of absorption decreases. In Table 2 we give a few values of $\Delta A_{n \mathrm{~s} / \mathrm{d} \rightarrow 1 \mathrm{~s}}\left(v_{\mathrm{c}}\right)$ for different frequencies $v_{\mathrm{c}}$. Given are the values of $\Delta A_{n \mathrm{~s} / \mathrm{d} \rightarrow 1 \mathrm{~s}}$ in $1 / \mathrm{s}$ for different frequencies $x_{\Gamma, \mathrm{c}}=\left[\nu_{\mathrm{c}}-v_{\alpha}\right] / \Gamma_{2 \mathrm{p} \rightarrow 1 \mathrm{~s}}$. In each column the first value is for the s-levels, the second for the d-states. 


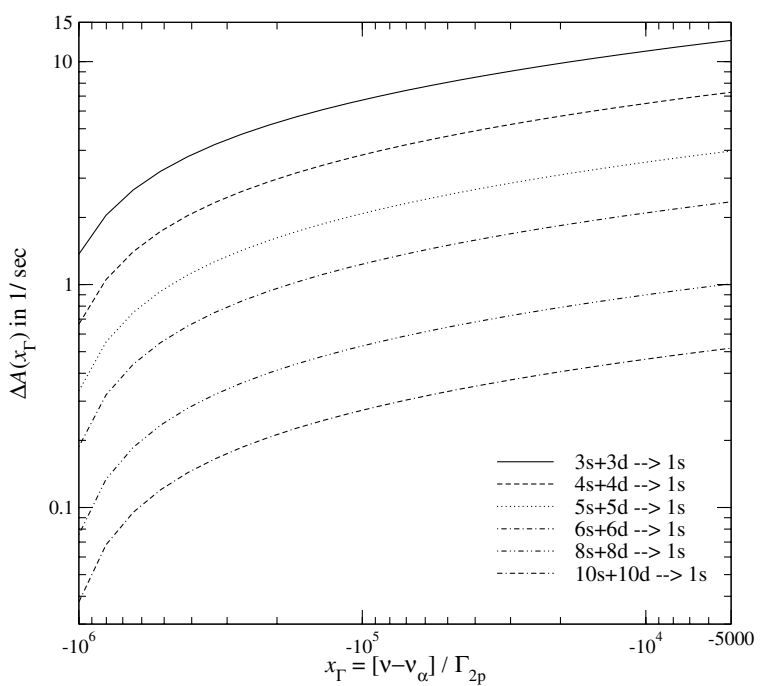

Fig. 10. Combined effective two-photon photon production rate, $\Delta A_{n \mathrm{~s}+n \mathrm{~d} \rightarrow 1 \mathrm{~s}}\left(v_{\mathrm{c}}\right)$, computed according to Eq. (26). To convert to the variable $\left[v-v_{\alpha}\right] / v_{\alpha}$, one should multiply $x_{\Gamma}$ by $\sim 2.54 \times 10^{-7}$.

Table 2. Effective difference in the photon production rate in the distant wings using Eq. (24).

\begin{tabular}{cccc}
\hline \hline$n$ & $x_{\Gamma, \mathrm{c}}=-10^{5}$ & $x_{\Gamma, \mathrm{c}}=-5 \times 10^{4}$ & $x_{\Gamma, \mathrm{c}}=-10^{4}$ \\
\hline 3 & $-0.307 / 8.133$ & $-0.951 / 9.882$ & $-2.565 / 13.883$ \\
4 & $-0.210 / 4.619$ & $-0.461 / 5.648$ & $-1.082 / 8.004$ \\
5 & $-0.122 / 2.523$ & $-0.245 / 3.082$ & $-0.551 / 4.356$ \\
6 & $-0.074 / 1.497$ & $-0.144 / 1.8272$ & $-0.317 / 2.578$ \\
7 & $-0.048 / 0.954$ & $-0.091 / 1.164$ & $-0.199 / 1.641$ \\
8 & $-0.033 / 0.643$ & $-0.061 / 0.785$ & $-0.133 / 1.106$ \\
9 & $-0.023 / 0.454$ & $-0.043 / 0.553$ & $-0.093 / 0.779$ \\
10 & $-0.017 / 0.331$ & $-0.032 / 0.404$ & $-0.068 / 0.569$ \\
\hline
\end{tabular}

Figure 10 also shows that, in contrast to the works of Dubrovich \& Grachev (2005) and Wong \& Scott (2007), the net photon escape rate due to the combined effect of the $s$ and d-state, two-photon process decreases with increasing $n$. This implies that the relevance of the two-photon emission from higher shells is significantly less than in their computations, because the sharp drop in the populations of levels with $n$ will no longer be partially canceled by the assumed linear increase in the effective two-photon-decay rate.

\subsubsection{Differences in the free electron fraction}

We modified our multi-level hydrogen code (for more details see Rubiño-Martín et al. 2006; Chluba et al. 2007) to take into account the additional escape of photons in the distant wings of the Lyman- $\alpha$ resonance due to the two-photon process using Eq. (25). For the hydrogen atom we typically included the first 30 shells in our computations, following the evolution of the populations for each angular-momentum substate separately. We also performed computations with more shells, but this did not alter the results significantly with our approach.

The additional two-photon process was included for $s$ and d-states with $n \leq n_{2 \gamma}$, where the parameter $n_{2 \gamma}$ gives the highest shell for which the additional two-photon decay was taken into account. We only used $n_{2 \gamma} \leq 10$, but because of the strong decrease of $\left|\Delta A_{n \mathrm{~s} / \mathrm{d} \rightarrow 1 \mathrm{~s}}\left(v_{\mathrm{c}}\right)\right|$ with $n$ (see Fig. 9) and the drop in the populations of higher shells, we do not expect any significant differences when going beyond this. For simplicity we also

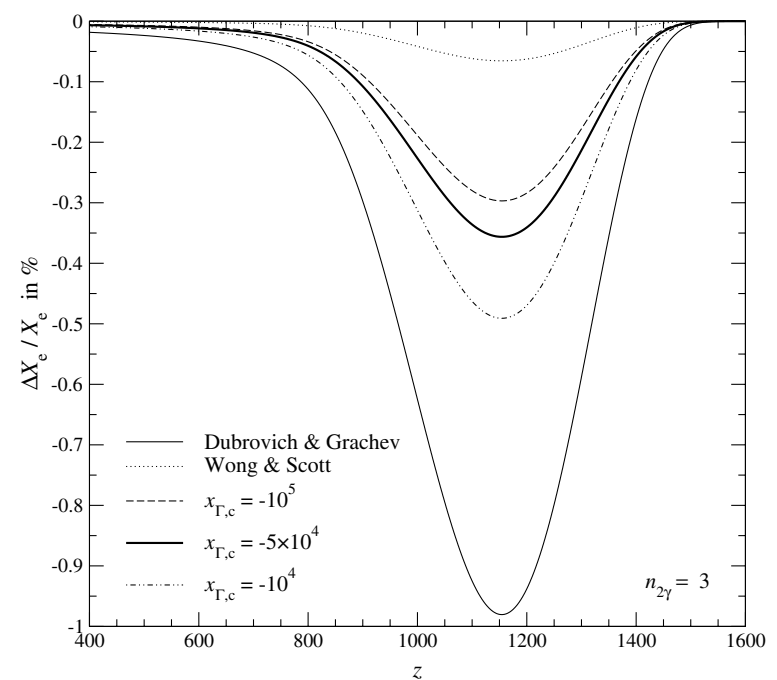

Fig. 11. Relative change in the free electron fraction. Here we only included the additional two-photon process for the $3 \mathrm{~s}$ and $3 \mathrm{~d}$-states. The computations were performed for a 30 -shell hydrogen atom. The effective two-photon rates for three different values of $v_{\mathrm{c}}$ according to Table 2 were used. For comparison, we show the results that were obtained using the $3 \mathrm{~s}$ and $3 \mathrm{~d}$ two-photon decay rates of Dubrovich \& Grachev (2005) and Wong \& Scott (2007).

assumed that the value of $v_{\mathrm{c}}$ is constant with time. This makes our estimates more conservative, since both at very low and very high redshifts, $v_{\mathrm{c}}$ should be closer to $v_{\alpha}$ and therefore may increase the impact of the two-photon process on the recombination history. We performed computations with three different values of $v_{\mathrm{c}}$. The effective rates for these cases are summarized in Table 2. We consider the case with $x_{\Gamma, \mathrm{c}}=-10^{5}$ as pessimistic, whereas the case $x_{\Gamma, \mathrm{c}}=-10^{4}$ may be optimistic.

We also ran computations using the formulae according to Dubrovich \& Grachev (2005) and Wong \& Scott (2007). In the paper of Dubrovich \& Grachev (2005), the s and d-rates were not given separately, but assuming $\Delta A_{n \mathrm{~s} \rightarrow 1 \mathrm{~s}}^{2 \gamma} \equiv \Delta A_{n \mathrm{~d} \rightarrow 1 \mathrm{~s}}^{2 \gamma}$ for simplicity, one finds

$\Delta A_{n \mathrm{~s} \rightarrow 1 \mathrm{~s}}^{2 \gamma, \mathrm{DG}} \equiv \Delta A_{n \mathrm{~d} \rightarrow 1 \mathrm{~s}}^{2 \gamma, \mathrm{DG}}=8.2293 \mathrm{~s}^{-1} \times S_{\mathrm{DG}}(n)$,

where $S_{\mathrm{DG}}(n)=9\left[\frac{n-1}{n+1}\right]^{2 n} \frac{11 n^{2}-41}{n}$. Since the deviations from full statistical equilibrium are rather small (Rubiño-Martín et al. 2006; Chluba et al. 2007), this assumption should not be very critical and, in any case, is only meant for comparison.

Wong \& Scott (2007) explicitly give the rates for the $3 \mathrm{~s}$ and $3 \mathrm{~d}$-states and then assume the same $n$-scaling as Dubrovich \& Grachev (2005). This yields

$\Delta A_{n \mathrm{~s} \rightarrow 1 \mathrm{~s}}^{2 \gamma, \mathrm{WS}}=8.2197 \mathrm{~s}^{-1} \times S_{\mathrm{WS}}(n)$
$\Delta A_{n \mathrm{~d} \rightarrow 1 \mathrm{~s}}^{2 \gamma, \mathrm{WS}}=0.13171 \mathrm{~s}^{-1} \times S_{\mathrm{WS}}(n)$,

with $S_{\mathrm{WS}}(n)=S_{\mathrm{DG}}(n) / S_{\mathrm{DG}}(3)=\frac{96}{29}\left[\frac{n-1}{n+1}\right]^{2 n} \frac{11 n^{2}-41}{n}$. Comparing with Eq. (27), one can see that the difference in the approach of Dubrovich \& Grachev (2005) and Wong \& Scott (2007) is mainly because they used a much lower rate for the d-states (a factor $\sim 170$ !). The assumed rate for the s-states is only $\sim 2.7$ times lower than in the computations of Dubrovich \& Grachev (2005).

In Fig. 11 we present the relative change in the free electron fraction when only including the additional two-photon process 


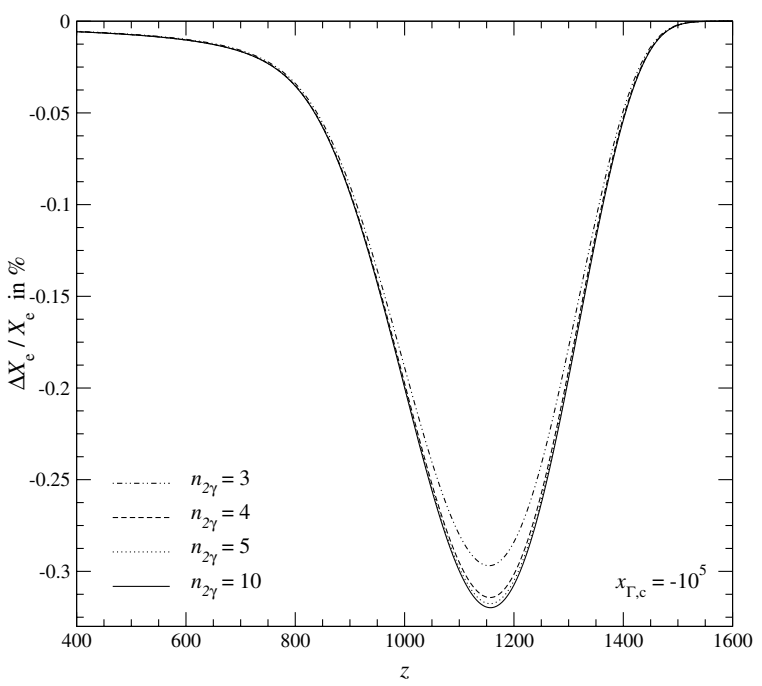

Fig. 12. Relative change in the free electron fraction for different values of $n_{2 \gamma}$. The computations were performed including 30 shells.

for $3 \mathrm{~s}$ and $3 \mathrm{~d}$-states. For comparison we show the results obtained using the decay rates of Dubrovich \& Grachev (2005) and Wong \& Scott (2007). One can clearly see that the dependence on the adopted value of $v_{\mathrm{c}}$ is not very strong. For our optimistic value of $v_{\mathrm{c}}$, close to the maximum the effect is roughly 2 times smaller than for the values of Dubrovich \& Grachev (2005), and even in our pessimistic model, it is still more than $\sim 4$ times greater than within the framework of Wong \& Scott (2007). Comparing the curves, which we obtained within the approach of Dubrovich \& Grachev (2005) and Wong \& Scott (2007), with those in Fig. 3 of Wong \& Scott (2007) one can see that our results for the changes in the electron fraction are slightly smaller. We checked that this is not due to our detailed treatment of the angular-momentum substates. This is expected since the deviations from full statistical equilibrium at the relevant redshifts are too small to have any effect here (Rubiño-Martín et al. 2006; Chluba et al. 2007). Also we computed the same correction using 50 shells, but found no significant increase.

In Fig. 12 we illustrate the impact of the two-photon process from higher shells. With our estimates of the effective twophoton decay rates, like in the studies of Dubrovich \& Grachev (2005) and Wong \& Scott (2007), the effect increases with $n_{2 \gamma}$. However, the strong decrease in the effective rates within our computations (see Table 2) implies that the result practically does not change when including the additional two-photon effect for more than 5 shells. This strongly contrasts the works of Dubrovich \& Grachev (2005) and Wong \& Scott (2007), where the total change in the free electron fraction radically depends on the chosen value of $n_{2 \gamma}$ (even up to $n_{2 \gamma}=40$ was considered). As mentioned above, in these computations the increase in the two-photon decay rates with $n$ (cf. Eqs. (27) and (28)) partially cancels the decrease in the population of the higher levels, and therefore enhances the impact of their contribution as compared to the lower shells. For example, at $z \sim 1200$ (i.e. close to the maximum of the changes in $N_{\mathrm{e}}$ ) the populations of the excited states are still nearly in Saha-equilibrium with the continuum (Chluba et al. 2007). Therefore the population of the fourth shell is roughly a factor of $\mathrm{e}^{-h v_{43} / k T_{\gamma}} \sim 0.19$ smaller than in the third shell. Also the effective $2 \gamma$-rate decreases by $\sim 1.8$, whereas in the picture of Dubrovich \& Grachev (2005) and Wong \& Scott (2007) it would have increased $\sim 1.9$ times. From Fig. 12 it is also clear that the strongest effect for our estimates of the

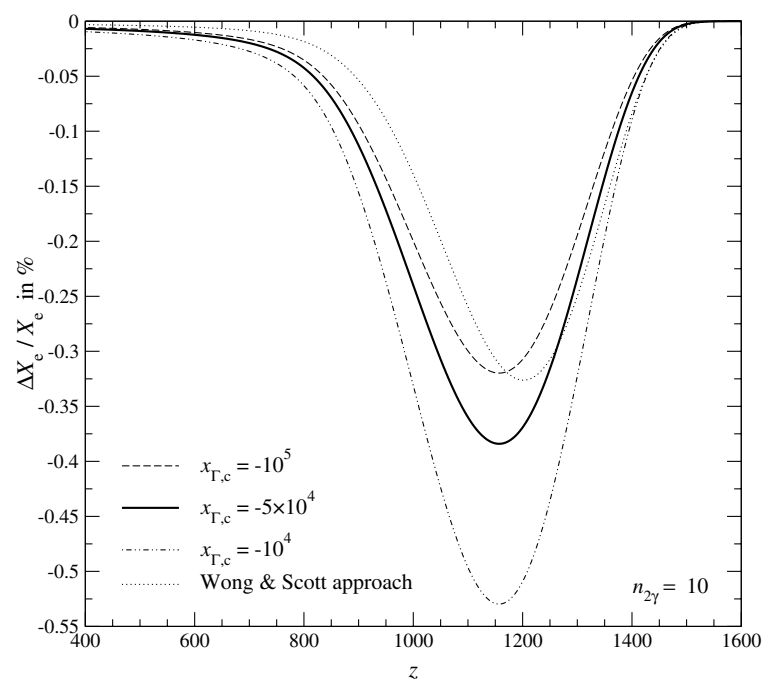

Fig. 13. Relative change in the free electron fraction when taking the additional two-photon emission for up to 10 shells into account. The computations were performed including 30 shells, for three different values of $v_{\mathrm{c}}$. For comparison, the result that was obtained within the approach of Wong \& Scott (2007) is shown, but for $n_{2 \gamma}=40$ and using a 50 -shell model for the hydrogen atom.

effective decay rates comes from the $3 \mathrm{~s}$ and $3 \mathrm{~d}$-levels alone. This again is in strong opposition to the computations of Dubrovich \& Grachev (2005) and Wong \& Scott (2007) where more than $\sim 75 \%$ of the correction is due to the combined effect of higher shells.

In Fig. 13 we give our final estimates for the possible changes in the recombination history. In our optimistic model the change is $\Delta X_{\mathrm{e}} / X_{\mathrm{e}} \sim-0.53 \%$ at redshift $z \sim 1150$, and it drops to $\Delta X_{\mathrm{e}} / X_{\mathrm{e}} \sim-0.32 \%$ for the pessimistic case. Including more shells in the model for the hydrogen atom did not change these results. For comparison we also computed the changes in the ionization history by applying the formulae of Wong \& Scott (2007), but using $n_{2 \gamma}=40$ and 50 shells for the model of the hydrogen atom. Although our discussion has shown that the values computed by Cresser et al. (1986) for 3s and 3d-states are likely not related to the cosmological hydrogen recombination problem and that extrapolating those values to higher shells is rather rough, our final results are numerically compatible with those obtained using the approach of Wong \& Scott (2007). However, within our approach the changes in the ionization history close to the maximum of the Thomson visibility function (Sunyaev \& Zeldovich 1970) are larger than in the computations of Wong $\&$ Scott (2007). Therefore the changes in the cosmic microwave background temperature and polarization power spectra are also expected to be a bit larger. Indeed it seems that the corrections to the ionization history due to the two-photon decay from higher shells does not reach the percent level, and that the impact of this process was overestimated by Dubrovich \& Grachev (2005).

\subsubsection{Additional remarks}

Here we have only investigated the bound-bound two-photon transitions directly leading to the ground state. Equations (1) and (6) are also applicable when the final state is any s-level. We also checked the rate for the two-photon transition $3 \mathrm{~s} \rightarrow 2 \mathrm{~s}$ and $3 \mathrm{~d} \rightarrow 2 \mathrm{~s}$ and, as expected, found very low values $\left(\sim 0.0885 \mathrm{~s}^{-1}\right.$ for the $3 \mathrm{~s}$ and $0.0278 \mathrm{~s}^{-1}$ in the case of $3 \mathrm{~d}$ ). In addition, because all dipole transitions to the second shell are optically thin 
in the recombination problem, these corrections should never be important within this context. Similarly, the $2 \mathrm{p} \rightarrow 1 \mathrm{~s}$ twophoton transition due to its low probability $\left(\sim\right.$ few $\times 10^{-6} \mathrm{~s}^{-1}$, see Labzowsky et al. 2005) can be completely ignored.

One may in addition consider the problem of two-photon transitions starting from the continuum, e.g. the recombination of electrons to the $2 \mathrm{p}$-state and subsequent release of a Lyman- $\alpha$ photon. Here deviations of the line profile from the normal Lorentzian shape can also be expected and may lead to an increase in the effective Lyman- $\alpha$ escape rate. However, since the supply of photons to the $2 p$-state by transitions from higher shells is several times faster, the total impact of this effect is very likely less than the one from the $2 \gamma$-transitions already discussed here.

As mentioned above, under physical conditions like those in our Universe during the epoch of hydrogen recombination, the coherence of two and possibly multi-photon processes is maintained. Consequently one should investigate how strong the deviations of the corresponding emission profiles from a Lorentzian are when more than two photons are involved. This requires a QED multi-photon treatment, which is beyond the scope of this paper. But as we have seen above, adding the two-photon process for the $4 \mathrm{~s}-$ and $4 \mathrm{~d}$-states (due to the drop in the population of these levels and decrease in their effective two-photon decay rate) has affected the recombination history at a level of $\sim 0.02 \%$ in addition to the $3 \mathrm{~s}$ and $3 \mathrm{~d}$ (see Fig. 12). For the $3 \gamma$-decay of the $4 \mathrm{f}-$ level, one expects that the relative correction will be close to the one from to the $3 \mathrm{~d}$ two-photon decay. This is because the largest term in this $3 \gamma$-description should involve at least one nearly resonant transition, since the other contributions should be suppressed in addition. For photons appearing close to the Lyman- $\alpha$ line, a nearly resonant $4 \mathrm{f} \rightarrow 3 \mathrm{~d}$ transition, followed by a quasi $2 \gamma$-decay of the $3 \mathrm{~d}$-state, is most likely. Therefore one has

$\Delta A_{4 \mathrm{f} \rightarrow 1 \mathrm{~s}}^{3 \gamma} \sim A_{4 \mathrm{f} \rightarrow 3 \mathrm{~d}}^{3 \gamma} \times \frac{\Delta A_{3 \mathrm{~d} \rightarrow 1 \mathrm{~s}}}{A_{3 \mathrm{~d} \rightarrow 1 \mathrm{~s}}^{2 \gamma}} \sim \frac{A_{4 \mathrm{f} \rightarrow 3 \mathrm{~d}}^{1 \gamma}}{A_{3 \mathrm{~d} \rightarrow 2 \mathrm{p}}^{1 \gamma}} \times \Delta A_{3 \mathrm{~d} \rightarrow 1 \mathrm{~s}}$,

where we have used ${ }^{11} A_{4 \mathrm{f} \rightarrow 3 \mathrm{~d}}^{3 \gamma} \sim A_{4 \mathrm{f} \rightarrow 3 \mathrm{~d}}^{1 \gamma}$ and $A_{3 \mathrm{~d} \rightarrow 2 \mathrm{p}}^{2 \gamma} \sim A_{3 \mathrm{~d} \rightarrow 2 \mathrm{p}}^{1 \gamma}$. With the value in Table 2, one finds $\Delta A_{4 \mathrm{f} \rightarrow 1 \mathrm{~s}}^{3 \gamma} \sim 2.1 \mathrm{~s}^{-1}$. Due to the statistical weight of the $4 \mathrm{f}$-state, one then should obtain another $\sim 7 \times 2.1 \mathrm{~s}^{-1} \sim 14.7 \mathrm{~s}^{-1}$ addition to the overall decay rate of the fourth level. This is roughly a $\sim 50 \%$ correction to the total two-photon correction of the fourth shell. Therefore, one could expect another $\sim 0.01 \%$ correction to the free electron faction when including the three-photon decay from the $4 \mathrm{f}$. Furthermore, for some high level (likely above $n \sim 20-50$ ), even the residual disturbances from the small amount of perturbers that are present in the Universe will destroy the coherence of the possible multi-photon emission coming from very high initial levels.

One could also consider the three-photon decay of the $2 p$-state. Here, just as in the 2s-two-photon decay, no intermediate resonances are involved; and due to momentum conservation, this process is allowed. However, simple estimates show that this process has a rate lower than $\sim 10^{-2} \mathrm{~s}^{-1}$ and hence negligible at the $0.1 \%$-level.

\section{Conclusions}

We have studied in detail the emission of photons due to twophoton transitions from high s and d-states to the ground level.

${ }^{11}$ In both cases the branching ratios are very close to unity.
Up to $n=20$ we found simple analytic fitting formulae to represent the full two-photon emission profile with very high accuracy. We have discussed the deviations in the two-photon emission profiles from the natural Lorentzian shape and investigated the importance of the non-resonant, cascade, and interference term separately.

Applying our results to the cosmological hydrogen recombination shows that the corrections to the ionization history due to the additional two-photon process from higher shells likely do not reach the percent level. For conservative assumptions we find a correction $\Delta X_{\mathrm{e}} / X_{\mathrm{e}} \sim-0.4 \%$ at redshift $z \sim 1160$. This is numerically similar to the result of Wong \& Scott (2007); however, the physics leading to this conclusion is rather different. In particular we find that the two-photon process for initial sstates actually slows the recombination process down. In addition, the effective two-photon rates connecting the high $\mathrm{s}$ and d-level directly to the 1s-level decrease with principle quantum number $n$. Both aspects contrast to the rate estimates used in the studies by Dubrovich \& Grachev (2005) and Wong \& Scott (2007). Here it is very important that the destructive interference between the cascade and non-resonant term cancels a large part of the additional non-resonant emission in the distant red wings of the Lyman- $\alpha$ transition. Furthermore, in our computations the main correction to the ionization history stems from the $3 \mathrm{~s}$ and 3d-states, while in the computations of Dubrovich \& Grachev (2005) and Wong \& Scott (2007) more than $\sim 75 \%$ of the correction is due to the combined effect of higher shells.

Acknowledgements. The authors are glad to thank S.G. Karshenboim for many useful discussions and consultations about details of the two-photon processes and for pointing us towards several useful references. They are also grateful to L. N. Labzowsky for his advice and detailed discussions of the two-photon emission. In particular J.C. thanks L. N. Labzowsky for hospitality during his visit in Dresden, December 2006. We also wish to thank S. G. Karshenboim and V.G. Ivanov for the possibility to compare our results with their computations on the $3 \mathrm{~s}$ and $3 \mathrm{~d}$ rates prior to publication. It was a pleasure to discuss the detailed physics of recombination with $\mathrm{C}$. Hirata during the visit to the IAS, September 2006. Furthermore the authors thank E. E. Kholupenko for his detailed comments on the paper.

\section{Appendix A: Radial integrals}

\section{A.1. Bound-bound radial integrals}

For the the required bound-bound radial integrals up to $n_{i}=5$, one has

$$
\begin{aligned}
\left\langle R_{1 \mathrm{~s}}|r| R_{n \mathrm{p}}\right\rangle= & 2^{4} n^{7 / 2} \frac{(n-1)^{n-5 / 2}}{(n+1)^{n+5 / 2}} \\
\left\langle R_{2 \mathrm{~s}}|r| R_{n \mathrm{p}}\right\rangle= & 2^{8} \sqrt{2} n^{7 / 2}\left(n^{2}-1\right)^{1 / 2} \frac{(n-2)^{n-3}}{(n+2)^{n+3}} \\
\left\langle R_{3 \mathrm{~s}}|r| R_{n \mathrm{p}}\right\rangle= & 2^{4} 3^{3} \sqrt{3} n^{7 / 2}\left(n^{2}-1\right)^{1 / 2} \\
& \times\left[7 n^{2}-27\right] \frac{(n-3)^{n-4}}{(n+3)^{n+4}}
\end{aligned}
$$

$\left\langle R_{4 \mathrm{~s}}|r| R_{n \mathrm{p}}\right\rangle=\frac{2^{13}}{3} n^{7 / 2}\left(n^{2}-1\right)^{1 / 2}$

$$
\times\left[23 n^{4}-288 n^{2}+768\right] \frac{(n-4)^{n-5}}{(n+4)^{n+5}}
$$




$$
\begin{aligned}
\left\langle R_{5 \mathrm{~s}}|r| R_{n \mathrm{p}}\right\rangle & =\frac{2^{4} 5^{4} \sqrt{5}}{3} n^{7 / 2}\left(n^{2}-1\right)^{1 / 2} \\
\times\left[91 n^{6}-\right. & \left.2545 n^{4}+20625 n^{2}-46875\right] \frac{(n-5)^{n-6}}{(n+5)^{n+6}} \\
\left\langle R_{3 \mathrm{~d}}|r| R_{n \mathrm{p}}\right\rangle= & \frac{2^{5} 3^{3} \sqrt{2} \sqrt{3}}{\sqrt{5}} n^{11 / 2}\left(n^{2}-1\right)^{1 / 2} \frac{(n-3)^{n-4}}{(n+3)^{n+4}} \\
\left\langle R_{4 \mathrm{~d}}|r| R_{n \mathrm{p}}\right\rangle= & \frac{2^{14}}{3 \sqrt{5}} n^{11 / 2}\left(n^{2}-1\right)^{1 / 2}\left[7 n^{2}-48\right] \frac{(n-4)^{n-5}}{(n+4)^{n+5}} \\
\left\langle R_{5 \mathrm{~d}}|r| R_{n \mathrm{p}}\right\rangle= & \frac{2^{5} 5^{4} \sqrt{2} \sqrt{5}}{3 \sqrt{7}} n^{11 / 2}\left(n^{2}-1\right)^{1 / 2} \\
& \times\left[29 n^{4}-590 n^{2}+2625\right] \frac{(n-5)^{n-6}}{(n+5)^{n+6}}
\end{aligned}
$$

In addition one needs $\left\langle R_{n, l-1}|r| R_{n l}\right\rangle=-\frac{3}{2} n \sqrt{n^{2}-l^{2}}$.

\section{A.2. Bound-free radial integrals}

For the necessary bound-free radial integrals up to $n_{i}=5$, using the definition of the radial functions for the continuum states (e.g. see Sect. 36, Landau 1977), one obtains

$$
\begin{aligned}
& \left\langle R_{1 \mathrm{~s}}|r| R_{x 1}\right\rangle=2^{4} \frac{x^{1 / 2}}{\left(1+x^{2}\right)^{5 / 2}} \frac{\mathrm{e}^{-2 \arctan (x) / x}}{\sqrt{1-\mathrm{e}^{-2 \pi / x}}} \\
& \left\langle R_{2 \mathrm{~s}}|r| R_{x 1}\right\rangle=2^{8} \sqrt{2} \frac{x^{1 / 2}\left(1+x^{2}\right)^{1 / 2}}{\left(1+4 x^{2}\right)^{3}} \frac{\mathrm{e}^{-2 \arctan (2 x) / x}}{\sqrt{1-\mathrm{e}^{-2 \pi / x}}} \\
& \left\langle R_{3 \mathrm{~s}}|r| R_{x 1}\right\rangle=2^{4} 3^{3} \sqrt{3} \frac{x^{1 / 2}\left(1+x^{2}\right)^{1 / 2}\left[7+27 x^{2}\right]}{\left(1+9 x^{2}\right)^{4}} \\
& \times \frac{\mathrm{e}^{-2 \arctan (3 x) / x}}{\sqrt{1-\mathrm{e}^{-2 \pi / x}}} \\
& \left\langle R_{4 \mathrm{~s}}|r| R_{x 1}\right\rangle=\frac{2^{13}}{3} \frac{x^{1 / 2}\left(1+x^{2}\right)^{1 / 2}\left[23+96 x^{2}\left(3+8 x^{2}\right)\right]}{\left(1+16 x^{2}\right)^{5}} \\
& \times \frac{\mathrm{e}^{-2 \arctan (4 x) / x}}{\sqrt{1-\mathrm{e}^{-2 \pi / x}}} \\
& \left\langle R_{5 \mathrm{~s}}|r| R_{x 1}\right\rangle=\frac{2^{4} 5^{4} \sqrt{5}}{3} \\
& \times \frac{x^{1 / 2}\left(1+x^{2}\right)^{1 / 2}\left[91+5 x^{2}\left(509+4125 x^{2}+9375 x^{4}\right)\right]}{\left(1+25 x^{2}\right)^{6}} \\
& \times \frac{\mathrm{e}^{-2 \arctan (5 x) / x}}{\sqrt{1-\mathrm{e}^{-2 \pi / x}}} \\
& \left\langle R_{3 \mathrm{~d}}|r| R_{x 1}\right\rangle=\frac{2^{5} 3^{3} \sqrt{2} \sqrt{3}}{\sqrt{5}} \frac{x^{1 / 2}\left(1+x^{2}\right)^{1 / 2}}{\left(1+9 x^{2}\right)^{4}} \frac{\mathrm{e}^{-2 \arctan (3 x) / x}}{\sqrt{1-\mathrm{e}^{-2 \pi / x}}} \\
& \left\langle R_{4 \mathrm{~d}}|r| R_{x 1}\right\rangle=\frac{2^{14}}{3 \sqrt{5}} \frac{x^{1 / 2}\left(1+x^{2}\right)^{1 / 2}\left[7+48 x^{2}\right]}{\left(1+16 x^{2}\right)^{5}} \\
& \times \frac{\mathrm{e}^{-2 \arctan (4 x) / x}}{\sqrt{1-\mathrm{e}^{-2 \pi / x}}}
\end{aligned}
$$$$
\left\langle R_{5 \mathrm{~d}}|r| R_{x 1}\right\rangle=\frac{2^{5} 5^{4} \sqrt{2} \sqrt{5}}{3 \sqrt{7}} \frac{x^{1 / 2}\left(1+x^{2}\right)^{1 / 2}\left[29+590 x^{2}+2625 x^{4}\right]}{\left(1+25 x^{2}\right)^{6}}
$$$$
\times \frac{\mathrm{e}^{-2 \arctan (5 x) / x}}{\sqrt{1-\mathrm{e}^{-2 \pi / x}}} .
$$

The value of $x$ ranges from 0 to $\infty$.

\section{References}

Akhiezer, A. I., \& Berestetskii, V. B. 1965, Quantum electrodynamics (John Wiley and Sons)

Berestetskii, V. B., Lifshits, E. M., \& Pitaevskii, L. P. 1982, Quantum electrodynamics, 2nd revised edition (Elsevier Butterworth Heinemann)

Breit, G., \& Teller, E. 1940, ApJ, 91, 215

Cesar, C. L., Fried, D. G., Killian, T. C., et al. 1996, Phys. Rev. Lett., 77, 255

Chluba, J., \& Sunyaev, R. A. 2006a, A\&A, 458, L29

Chluba, J., \& Sunyaev, R. A. 2006b, A\&A, 446, 39

Chluba, J., \& Sunyaev, R. A. 2007a, in preparation

Chluba, J., \& Sunyaev, R. A. 2007b, A\&A, 475, 109

Chluba, J., Rubiño-Martín, J. A., \& Sunyaev, R. A. 2007, MNRAS, 374, 1310

Cocke, C. L., Curnutte, B., MacDonald, J. R., Bedner, J. A., \& Marrus, R. 1974, Phys. Rev. A, 9, 2242

Cresser, J. D., Tang, A. Z., Salamo, G. J., \& Chan, F. T. 1986, Phys. Rev. A, 33, 1677

Drake, G. W. F. 1986, Phys. Rev. A, 34, 2871

Dubrovich, V. K. 1987, Opt. Spectr., 63, 256

Dubrovich, V. K., \& Grachev, S. I. 2005, Astron. Lett., 31, 359

Fixsen, D. J., \& Mather, J. C. 2002, ApJ, 581, 817

Florescu, V. 1984, Phys. Rev. A, 30, 2441

Florescu, V., Schneider, I., \& Mihailescu, I. N. 1988, Phys. Rev. A, 38, 2189

Goldman, S. P. 1989, Phys. Rev. A, 40, 1185

Goldman, S. P., \& Drake, G. W. F. 1981, Phys. Rev. A, 24, 183

Göppert-Mayer 1931, Ann. Phys., 9, 273

Gould, H., \& Marrus, R. 1983, Phys. Rev. A, 28, 2001

Grachev, S. I., \& Dubrovich, V. K. 1991, Astrophysics, 34, 124

Hinds, E. A., Clendenin, J. E., \& Novick, R. 1978, Phys. Rev. A, 17, 670

Hinshaw, G., Nolta, M. R., Bennett, C. L., et al. 2006, ArXiv Astrophysics e-prints

Hirata, C. M., \& Switzer, E. R. 2007, ArXiv Astrophysics e-prints

Johnson, W. R. 1972, Phys. Rev. Lett., 29, 1123

Karshenboim, S. G., \& Ivanov, V. G. 2007, Astron. Lett., submitted

Kholupenko, E. E., \& Ivanchik, A. V. 2006, Astron. Lett., 32, 795

Kholupenko, E. E., Ivanchik, A. V., \& Varshalovich, D. A. 2007, ArXiv Astrophysics e-prints

Kipper, A. Y. 1950, Astron. Zh., 27, 321

Klarsfeld, S. 1969, Phys. Lett. A, 30, 382

Kocher, C. A., Clendenin, J. E., \& Novick, R. 1972, Phys. Rev. Lett., 29, 615

Krüger, H., \& Oed, A. 1975, Phys. Lett. A, 54, 251

Labzowsky, L. N., \& Shonin, A. V. 2004, Phys. Rev. A, 69, 012503

Labzowsky, L. N., Shonin, A. V., \& Solovyev, D. A. 2005, J. Phys. B Atom. Mol. Phys., 38, 265

Landau, L. D., \& Lifshits, E. M. 1977, Quantum Mechanics, 3nd revised edition (Butterworth-Heinemann)

Lewis, A., Weller, J., \& Battye, R. 2006 [arXiv:astro-ph/0606552]

Low, F. 1952, Phys. Rev., 88, 53

Marrus, R., \& Schmieder, R. W. 1972, Phys. Rev. A, 5, 1160

Mihalas, D. 1978, Stellar atmospheres, 2nd edn. (San Francisco, W. H. Freeman and Co.), 650

Novosyadlyj, B. 2006, MNRAS, 370, 1771

Page, L., Hinshaw, G., Komatsu, E., et al. 2006, ArXiv Astrophysics e-prints

Peebles, P. J. E. 1968, ApJ, 153, 1

Prior, M. H. 1972, Phys. Rev. Lett., 29, 611

Quattropani, A., Bassani, F., \& Carillo, S. 1982, Phys. Rev. A, 25, 3079

Rubiño-Martín, J. A., Chluba, J., \& Sunyaev, R. A. 2006, MNRAS, 371, 1939

Rybicki, G. B., \& dell'Antonio, I. P. 1994, ApJ, 427, 603

Seager, S., Sasselov, D. D., \& Scott, D. 1999, ApJ, 523, L1

Seager, S., Sasselov, D. D., \& Scott, D. 2000, ApJS, 128, 407

Seaton, M. J. 1959, MNRAS, 119, 90

Sobelman, I. I., Vainshtein, L. A., \& Yukov, E. A. 1995, Excitation of Atoms and Broadening of Spectral Lines, X (Berlin, Heidelberg, New York: SpringerVerlag), also Springer Series on Atoms and Plasmas, 15

Spitzer, L. J., \& Greenstein, J. L. 1951, ApJ, 114, 407

Storey, P. J., \& Hummer, D. G. 1991, Computer Phys. Commun., 66, 129

Sunyaev, R. A., \& Zeldovich, Y. B. 1970, Ap\&SS, 7, 3

Switzer, E. R., \& Hirata, C. M. 2007a, ArXiv Astrophysics e-prints

Switzer, E. R., \& Hirata, C. M. 2007b, ArXiv Astrophysics e-prints

Tung, J. H., Ye, X. M., Salamo, G. J., \& Chan, F. T. 1984, Phys. Rev. A, 30, 1175

Varshalovich, D. A., \& Syunyaev, R. A. 1968, Astrophysics, 4, 140

Weisskopf, V. 1932, Z. Phys., 75, 287

Weisskopf, V., \& Wigner, E. 1930, Z. Phys., 63, 54

Wong, W. Y., \& Scott, D. 2007, MNRAS, 375, 1441

Zeldovich, Y. B., Kurt, V. G., \& Syunyaev, R. A. 1968, Zh. Eksper. Teor. Fiziki, 55,278 
J. Chluba and R. A. Sunyaev: Two-photon transitions in hydrogen and cosmological recombination, Online Material p 1

\section{Appendix B: Non-linear fitting functions \\ non-resonant emission spectra}

Tables B.1 and B.2 contain the non-linear fitting coefficients for the non-resonant emission spectra. The non-resonant emission spectra are then given by $\phi_{n_{i} l_{i} \rightarrow 1 \mathrm{~s}}^{\mathrm{nr}}(y)=\sigma(y)^{2}$, with $\sigma(y)=$ $w^{1 / 2}\left[a_{0}+b_{0} w^{\beta}\left(1+b_{1} w^{1 / 8}+b_{2} w^{1 / 7}+b_{3} w^{1 / 6}+b_{4} w^{1 / 5}+b_{5} w^{1 / 4}+\right.\right.$ $\left.\left.b_{6} w^{1 / 3}+b_{7} w^{1 / 2}+b_{8} w^{1}+b_{9} w^{2}\right)\right]$ and $w=y(1-y)$. In this definition one has $M_{\mathrm{nr}}=\sigma(y) / \sqrt{G_{n_{i} l_{i}}} w^{3 / 2}$. The first 200 terms in the infinite sum were taken into account. Within the assumptions, the accuracy of these approximations should be better than $\sim 0.1 \%$. Note that $a_{0}$ and $b_{0}$ have dimension $\mathrm{s}^{-1 / 2}$, and $\phi^{\mathrm{nr}}(1 / 2)$ has dimension $\mathrm{s}^{-1}$. 
J. Chluba and R. A. Sunyaev: Two-photon transitions in hydrogen and cosmological recombination, Online Material p 2

Table B.1. Non-linear fitting coefficients for the non-resonant $n \mathrm{~s} \rightarrow 1$ s emission spectra within the frequency range $10^{-3} \leq y \leq 0.999$ for $n \leq 20$.

\begin{tabular}{|c|c|c|c|c|c|}
\hline & $2 \mathrm{~s}$ & $3 \mathrm{~s}$ & $\overline{4 s}$ & 5s & $\overline{6 s}$ \\
\hline$a_{0}$ & $-1.3974426528 \times 10^{1}$ & $-2.0848318929 \times 10^{1}$ & $-2.5412664413 \times 10^{1}$ & $-2.8856705303 \times 10^{1}$ & $-3.1567507558 \times 10^{1}$ \\
\hline$\beta$ & $2.5591291935 \times 10^{-1}$ & $5.5198239644 \times 10^{-1}$ & $8.0397418838 \times 10^{-1}$ & $4.8110524091 \times 10^{-1}$ & $2.5266888083 \times 10^{-1}$ \\
\hline$b_{0}$ & $6.6487585307 \times 10^{0}$ & $4.6179953215 \times 10^{2}$ & $7.6795669026 \times 10^{2}$ & $8.3598144011 \times 10^{2}$ & $5.6902488765 \times 10^{2}$ \\
\hline$b_{1}$ & $-5.1211954644 \times 10^{1}$ & $-9.8891531378 \times 10^{1}$ & $-4.2588083735 \times 10^{2}$ & $5.1404483119 \times 10^{1}$ & $4.5519082179 \times 10^{1}$ \\
\hline$b_{2}$ & $3.4686628383 \times 10^{1}$ & $1.0769127960 \times 10^{2}$ & $7.0510361599 \times 10^{2}$ & $-7.2853184204 \times 10^{1}$ & $-6.7551149725 \times 10^{1}$ \\
\hline$b_{3}$ & $3.8515432953 \times 10^{1}$ & $6.1301937755 \times 10^{1}$ & $-3.6120946105 \times 10^{1}$ & $-4.9378611706 \times 10^{1}$ & $-3.8327617310 \times 10^{1}$ \\
\hline$b_{4}$ & $6.7503966084 \times 10^{0}$ & $-4.1466090643 \times 10^{1}$ & $-4.4049919721 \times 10^{2}$ & $4.7383284338 \times 10^{1}$ & $4.4401360958 \times 10^{1}$ \\
\hline$b_{5}$ & $-3.1431622488 \times 10^{1}$ & $-8.4016250015 \times 10^{1}$ & $2.4657329140 \times 10^{2}$ & $9.9191282175 \times 10^{1}$ & $7.4195208626 \times 10^{1}$ \\
\hline$b_{6}$ & $-1.3697636757 \times 10^{1}$ & $7.1191755771 \times 10^{1}$ & $-6.5900037717 \times 10^{1}$ & $-1.0379836854 \times 10^{2}$ & $-7.9312304169 \times 10^{1}$ \\
\hline$b_{7}$ & $2.4016413274 \times 10^{1}$ & $-1.8695504961 \times 10^{1}$ & $1.8226732411 \times 10^{1}$ & $2.9602921680 \times 10^{1}$ & $2.1866188936 \times 10^{1}$ \\
\hline$b_{8}$ & $-9.5149995633 \times 10^{0}$ & $2.2794449683 \times 10^{0}$ & $-2.8692508508 \times 10^{0}$ & $-2.8636914451 \times 10^{0}$ & $-1.9806370670 \times 10^{0}$ \\
\hline$b_{9}$ & $2.6555412799 \times 10^{0}$ & $-4.3875761781 \times 10^{-1}$ & $4.4042663362 \times 10^{-1}$ & $3.8579054257 \times 10^{-1}$ & $2.6143991878 \times 10^{-1}$ \\
\hline \multirow[t]{2}{*}{$\phi^{\mathrm{nr}}(1 / 2)$} & $2.1303295046 \times 10^{1}$ & $2.5955835234 \times 10^{1}$ & $3.0008781647 \times 10^{1}$ & $3.5729374807 \times 10^{1}$ & $4.2778194752 \times 10^{1}$ \\
\hline & $7 \mathrm{~s}$ & $8 \mathrm{~s}$ & $9 \mathrm{~s}$ & $10 \mathrm{~s}$ & $11 \mathrm{~s}$ \\
\hline$a_{0}$ & $-3.3720076517 \times 10^{1}$ & $-3.5420358274 \times 10^{1}$ & $-3.6749872667 \times 10^{1}$ & $-3.7779791307 \times 10^{1}$ & $-3.8573952826 \times 10^{1}$ \\
\hline$\beta$ & $8.5151589950 \times 10^{-2}$ & $-4.2719713121 \times 10^{-2}$ & $-1.3118006971 \times 10^{-1}$ & $-2.1932912263 \times 10^{-1}$ & $-2.9226372396 \times 10^{-1}$ \\
\hline$b_{0}$ & $3.7656038819 \times 10^{2}$ & $2.7408956380 \times 10^{2}$ & $2.8136510570 \times 10^{2}$ & $1.5264948382 \times 10^{2}$ & $8.6437136666 \times 10^{1}$ \\
\hline$b_{1}$ & $2.7980511952 \times 10^{1}$ & $7.4703207553 \times 10^{0}$ & $-1.8981579892 \times 10^{1}$ & $-1.9235840320 \times 10^{1}$ & $-1.9681933480 \times 10^{1}$ \\
\hline$b_{2}$ & $-4.9359948916 \times 10^{1}$ & $-2.6259765669 \times 10^{1}$ & $6.6356770418 \times 10^{0}$ & $7.1592304894 \times 10^{0}$ & $7.8432338862 \times 10^{0}$ \\
\hline$b_{3}$ & $-2.0507457345 \times 10^{1}$ & $-3.3913231244 \times 10^{0}$ & $1.3837014244 \times 10^{1}$ & $1.3979846789 \times 10^{1}$ & $1.4343371719 \times 10^{1}$ \\
\hline$b_{4}$ & $3.6291414100 \times 10^{1}$ & $2.5464049259 \times 10^{1}$ & $7.8618686503 \times 10^{0}$ & $7.3730033289 \times 10^{0}$ & $6.8679571684 \times 10^{0}$ \\
\hline$b_{5}$ & $4.3713955958 \times 10^{1}$ & $1.6166653183 \times 10^{1}$ & $-8.0358407914 \times 10^{0}$ & $-8.6532015972 \times 10^{0}$ & $-9.6695240617 \times 10^{0}$ \\
\hline$b_{6}$ & $-5.2721158916 \times 10^{1}$ & $-2.8335792233 \times 10^{1}$ & $-4.8006902023 \times 10^{0}$ & $-3.6779369765 \times 10^{0}$ & $-2.2948815920 \times 10^{0}$ \\
\hline$b_{7}$ & $1.4831730999 \times 10^{1}$ & $8.6649789093 \times 10^{0}$ & $2.8424870765 \times 10^{0}$ & $2.4362978935 \times 10^{0}$ & $2.0390764869 \times 10^{0}$ \\
\hline$b_{8}$ & $-1.3230829528 \times 10^{0}$ & $-7.9156543417 \times 10^{-1}$ & $-3.2207517000 \times 10^{-1}$ & $-2.7645228408 \times 10^{-1}$ & $-2.3641043114 \times 10^{-1}$ \\
\hline$b_{9}$ & $1.7474071394 \times 10^{-1}$ & $1.0596166967 \times 10^{-1}$ & $4.6227764509 \times 10^{-2}$ & $3.9732841793 \times 10^{-2}$ & $3.4157538873 \times 10^{-2}$ \\
\hline \multirow[t]{2}{*}{$\phi^{\mathrm{nr}}(1 / 2)$} & $5.0793294231 \times 10^{1}$ & $5.9525977879 \times 10^{1}$ & $6.8805689403 \times 10^{1}$ & $7.8512293219 \times 10^{1}$ & $8.8558644431 \times 10^{1}$ \\
\hline & $12 \mathrm{~s}$ & $13 \mathrm{~s}$ & $14 \mathrm{~s}$ & $15 \mathrm{~s}$ & $16 \mathrm{~s}$ \\
\hline$a_{0}$ & $-3.9188326323 \times 10^{1}$ & $-3.9670102478 \times 10^{1}$ & $-4.0057506143 \times 10^{1}$ & $-4.0380409686 \times 10^{1}$ & $-4.0661454371 \times 10^{1}$ \\
\hline$\beta$ & $-3.5484078761 \times 10^{-1}$ & $-4.0844617686 \times 10^{-1}$ & $-4.5428570381 \times 10^{-1}$ & $-4.6335483910 \times 10^{-1}$ & $-4.9050805652 \times 10^{-1}$ \\
\hline$b_{0}$ & $4.9431541043 \times 10^{1}$ & $2.9197464029 \times 10^{1}$ & $1.7971514946 \times 10^{1}$ & $1.5982980641 \times 10^{1}$ & $1.1724743879 \times 10^{1}$ \\
\hline$b_{1}$ & $-1.9750664049 \times 10^{1}$ & $-1.9723076238 \times 10^{1}$ & $-1.9651279272 \times 10^{1}$ & $-2.0606406024 \times 10^{1}$ & $-2.1081283167 \times 10^{1}$ \\
\hline$b_{2}$ & $8.1838767684 \times 10^{0}$ & $8.3673624537 \times 10^{0}$ & $8.4788048970 \times 10^{0}$ & $9.3338234414 \times 10^{0}$ & $9.8388495127 \times 10^{0}$ \\
\hline$b_{3}$ & $1.4326519992 \times 10^{1}$ & $1.4291867041 \times 10^{1}$ & $1.4206704409 \times 10^{1}$ & $1.4985604253 \times 10^{1}$ & $1.5395638329 \times 10^{1}$ \\
\hline$b_{4}$ & $6.4270125576 \times 10^{0}$ & $6.0967371906 \times 10^{0}$ & $5.8366404588 \times 10^{0}$ & $5.7371156374 \times 10^{0}$ & $5.5633379574 \times 10^{0}$ \\
\hline$b_{5}$ & $-1.0032296167 \times 10^{1}$ & $-1.0363858125 \times 10^{1}$ & $-1.0518387038 \times 10^{1}$ & $-1.1546724399 \times 10^{1}$ & $-1.2242342508 \times 10^{1}$ \\
\hline$b_{6}$ & $-1.4087194335 \times 10^{0}$ & $-6.8184121010 \times 10^{-1}$ & $-2.9722754662 \times 10^{-1}$ & $1.1575092351 \times 10^{-1}$ & $4.8942663430 \times 10^{-1}$ \\
\hline$b_{7}$ & $1.8482968593 \times 10^{0}$ & $1.8529967851 \times 10^{0}$ & $2.1519310722 \times 10^{0}$ & $2.2891230659 \times 10^{0}$ & $2.6782065008 \times 10^{0}$ \\
\hline$b_{8}$ & $-2.1185600113 \times 10^{-1}$ & $-1.9498101086 \times 10^{-1}$ & $-1.8067549402 \times 10^{-1}$ & $-2.0119354736 \times 10^{-1}$ & $-1.8223251218 \times 10^{-1}$ \\
\hline & $3.0555243512 \times 10^{-2}$ & $2.7915669075 \times 10^{-2}$ & $2.5650331467 \times 10^{-2}$ & $3.2820939449 \times 10^{-2}$ & $3.0707648484 \times 10^{-2}$ \\
\hline \multirow[t]{2}{*}{$\phi^{\mathrm{nr}}(1 / 2)$} & $9.8879969305 \times 10^{1}$ & $1.0942707459 \times 10^{2}$ & $1.2016192323 \times 10^{2}$ & $1.3105436054 \times 10^{2}$ & $1.4208118814 \times 10^{2}$ \\
\hline & $17 \mathrm{~s}$ & $18 \mathrm{~s}$ & $19 \mathrm{~s}$ & $20 \mathrm{~s}$ & \\
\hline$\overline{a_{0}}$ & $-4.0917355907 \times 10^{1}$ & $-4.1160166944 \times 10^{1}$ & $-4.1398378313 \times 10^{1}$ & $-4.1637819958 \times 10^{1}$ & \\
\hline$\beta$ & $-4.7538949275 \times 10^{-1}$ & $-4.4937224788 \times 10^{-1}$ & $-4.4703263317 \times 10^{-1}$ & $-4.8244405309 \times 10^{-1}$ & \\
\hline$b_{0}$ & $1.2547183851 \times 10^{1}$ & $1.4317441971 \times 10^{1}$ & $1.3339226887 \times 10^{1}$ & $9.1516183240 \times 10^{0}$ & \\
\hline$b_{1}$ & $-2.1972421315 \times 10^{1}$ & $-2.2875778234 \times 10^{1}$ & $-2.3839004652 \times 10^{1}$ & $-2.3934619536 \times 10^{1}$ & \\
\hline$b_{2}$ & $1.0636513367 \times 10^{1}$ & $1.1277442747 \times 10^{1}$ & $1.2219984677 \times 10^{1}$ & $1.2479746639 \times 10^{1}$ & \\
\hline$b_{3}$ & $1.5943304131 \times 10^{1}$ & $1.6640858983 \times 10^{1}$ & $1.7332324934 \times 10^{1}$ & $1.7384969031 \times 10^{1}$ & \\
\hline$b_{4}$ & $5.4874376556 \times 10^{0}$ & $5.6104613925 \times 10^{0}$ & $5.3841616235 \times 10^{0}$ & $5.1298737644 \times 10^{0}$ & \\
\hline$b_{5}$ & $-1.2655779187 \times 10^{1}$ & $-1.3260181912 \times 10^{1}$ & $-1.4146776145 \times 10^{1}$ & $-1.4462821999 \times 10^{1}$ & \\
\hline$b_{6}$ & $4.9579530934 \times 10^{-1}$ & $5.2627269447 \times 10^{-1}$ & $1.0461078949 \times 10^{0}$ & $1.3716208664 \times 10^{0}$ & \\
\hline$b_{7}$ & $2.5916598871 \times 10^{0}$ & $2.4342837775 \times 10^{0}$ & $2.3937323753 \times 10^{0}$ & $2.8470359883 \times 10^{0}$ & \\
\hline$b_{8}$ & $-2.2938505101 \times 10^{-1}$ & $-2.7807376757 \times 10^{-1}$ & $-2.8519500457 \times 10^{-1}$ & $-2.4816096711 \times 10^{-1}$ & \\
\hline$b_{9}$ & $4.2155577449 \times 10^{-2}$ & $5.4040208750 \times 10^{-2}$ & $5.6731652666 \times 10^{-2}$ & $4.9205565009 \times 10^{-2}$ & \\
\hline$\phi^{\mathrm{nr}}(1 / 2)$ & $1.5322255378 \times 10^{2}$ & $1.6446312975 \times 10^{2}$ & $1.7579037822 \times 10^{2}$ & $1.8719377215 \times 10^{2}$ & \\
\hline
\end{tabular}


J. Chluba and R. A. Sunyaev: Two-photon transitions in hydrogen and cosmological recombination, Online Material p 3

Table B.2. Non-linear fitting coefficients for the non-resonant $n \mathrm{~d} \rightarrow 1$ s emission spectra within the frequency range $10^{-3} \leq y \leq 0.999$ for $n \leq 20$.

\begin{tabular}{|c|c|c|c|c|c|}
\hline & $\overline{3 d}$ & $\overline{4 d \mathrm{~d}}$ & $\overline{5 \mathrm{~d}}$ & $\overline{6 \mathrm{~d}}$ & $\overline{7 d}$ \\
\hline$a_{0}$ & $-1.0484392971 \times 10^{1}$ & $-1.4527047719 \times 10^{1}$ & $-1.7350074013 \times 10^{1}$ & $-1.9527421713 \times 10^{1}$ & $-2.1271595034 \times 10^{1}$ \\
\hline$\beta$ & $2.6115596268 \times 10^{-1}$ & $8.1050542323 \times 10^{-1}$ & $4.6206291704 \times 10^{-1}$ & $2.3419462081 \times 10^{-1}$ & $6.7999906938 \times 10^{-2}$ \\
\hline$b_{0}$ & $5.3546036998 \times 10^{1}$ & $1.7065087104 \times 10^{2}$ & $2.0902445563 \times 10^{2}$ & $1.4535416639 \times 10^{2}$ & $1.1921878150 \times 10^{2}$ \\
\hline$b_{1}$ & $-9.7319568527 \times 10^{1}$ & $-4.2936500531 \times 10^{2}$ & $5.8562543507 \times 10^{1}$ & $5.9612688683 \times 10^{1}$ & $3.0991642200 \times 10^{1}$ \\
\hline$b_{2}$ & $1.0624929126 \times 10^{2}$ & $7.1072286522 \times 10^{2}$ & $-7.8501054392 \times 10^{1}$ & $-8.0969461685 \times 10^{1}$ & $-5.2470184998 \times 10^{1}$ \\
\hline$b_{3}$ & $6.0569007312 \times 10^{1}$ & $-3.6472867939 \times 10^{1}$ & $-5.8200368181 \times 10^{1}$ & $-5.3437128442 \times 10^{1}$ & $-2.3362093646 \times 10^{1}$ \\
\hline$b_{4}$ & $-4.1091307521 \times 10^{1}$ & $-4.4275350080 \times 10^{2}$ & $4.8846690629 \times 10^{1}$ & $4.9735078265 \times 10^{1}$ & $3.7636220223 \times 10^{1}$ \\
\hline$b_{5}$ & $-8.3346792794 \times 10^{1}$ & $2.4703670467 \times 10^{2}$ & $1.1281858951 \times 10^{2}$ & $9.8247844915 \times 10^{1}$ & $4.8276486304 \times 10^{1}$ \\
\hline$b_{6}$ & $6.9608223453 \times 10^{1}$ & $-6.6433743910 \times 10^{1}$ & $-1.1364879196 \times 10^{2}$ & $-9.8596391243 \times 10^{1}$ & $-5.6611227459 \times 10^{1}$ \\
\hline$b_{7}$ & $-1.7046798510 \times 10^{1}$ & $1.8959198585 \times 10^{1}$ & $3.1841053645 \times 10^{1}$ & $2.6560347162 \times 10^{1}$ & $1.5852011862 \times 10^{1}$ \\
\hline$b_{8}$ & $1.6052249571 \times 10^{0}$ & $-3.1192847294 \times 10^{0}$ & $-3.0563352329 \times 10^{0}$ & $-2.3898992832 \times 10^{0}$ & $-1.4245686402 \times 10^{0}$ \\
\hline$b_{9}$ & $-2.2422235582 \times 10^{-1}$ & $5.0786391006 \times 10^{-1}$ & $4.1302480839 \times 10^{-1}$ & $3.1693775497 \times 10^{-1}$ & $1.8991428606 \times 10^{-1}$ \\
\hline \multirow[t]{2}{*}{$\phi^{\mathrm{nr}}(1 / 2)$} & $2.0915177571 \times 10^{1}$ & $3.4902680010 \times 10^{1}$ & $4.4901877444 \times 10^{1}$ & $5.2879363207 \times 10^{1}$ & $5.9763238933 \times 10^{1}$ \\
\hline & $8 \mathrm{~d}$ & $9 \mathrm{~d}$ & $10 \mathrm{~d}$ & $11 d$ & $12 \mathrm{~d}$ \\
\hline$a_{0}$ & $-2.2687581599 \times 10^{1}$ & $-2.3840999089 \times 10^{1}$ & $-2.4781138994 \times 10^{1}$ & $-2.5548956191 \times 10^{1}$ & $-2.6179506753 \times 10^{1}$ \\
\hline$\beta$ & $-4.0416246997 \times 10^{-2}$ & $-1.4739834639 \times 10^{-1}$ & $-2.3472013799 \times 10^{-1}$ & $-3.0648469505 \times 10^{-1}$ & $-3.6629270128 \times 10^{-1}$ \\
\hline$b_{0}$ & $1.8805941117 \times 10^{2}$ & $1.0632962733 \times 10^{2}$ & $6.0922960536 \times 10^{1}$ & $3.6160368635 \times 10^{1}$ & $2.2144091289 \times 10^{1}$ \\
\hline$b_{1}$ & $-1.8483714555 \times 10^{1}$ & $-1.8910499138 \times 10^{1}$ & $-1.9083232938 \times 10^{1}$ & $-1.9357145527 \times 10^{1}$ & $-1.9508239494 \times 10^{1}$ \\
\hline$b_{2}$ & $5.8156915967 \times 10^{0}$ & $6.5026790075 \times 10^{0}$ & $6.8739045830 \times 10^{0}$ & $7.3456517995 \times 10^{0}$ & $7.6357892226 \times 10^{0}$ \\
\hline$b_{3}$ & $1.3504738304 \times 10^{1}$ & $1.3795221365 \times 10^{1}$ & $1.3924013399 \times 10^{1}$ & $1.4140788681 \times 10^{1}$ & $1.4314213871 \times 10^{1}$ \\
\hline$b_{4}$ & $8.5177417832 \times 10^{0}$ & $8.0023959097 \times 10^{0}$ & $7.6277097156 \times 10^{0}$ & $7.2310576094 \times 10^{0}$ & $6.9389854602 \times 10^{0}$ \\
\hline$b_{5}$ & $-7.0817594819 \times 10^{0}$ & $-7.9116375657 \times 10^{0}$ & $-8.4667321100 \times 10^{0}$ & $-9.1736549163 \times 10^{0}$ & $-9.8020559467 \times 10^{0}$ \\
\hline$b_{6}$ & $-6.3232881999 \times 10^{0}$ & $-5.0886630492 \times 10^{0}$ & $-4.1097110628 \times 10^{0}$ & $-3.0436236228 \times 10^{0}$ & $-2.1203514774 \times 10^{0}$ \\
\hline$b_{7}$ & $3.4340616701 \times 10^{0}$ & $2.9768149183 \times 10^{0}$ & $2.6171546155 \times 10^{0}$ & $2.2949189350 \times 10^{0}$ & $2.0887633671 \times 10^{0}$ \\
\hline$b_{8}$ & $-3.9664209528 \times 10^{-1}$ & $-3.4089403622 \times 10^{-1}$ & $-2.9766246686 \times 10^{-1}$ & $-2.6063687833 \times 10^{-1}$ & $-2.3211600328 \times 10^{-1}$ \\
\hline$b_{9}$ & $5.7497854559 \times 10^{-2}$ & $4.9213789949 \times 10^{-2}$ & $4.2727779792 \times 10^{-2}$ & $3.7171055385 \times 10^{-2}$ & $3.2750870685 \times 10^{-2}$ \\
\hline \multirow[t]{2}{*}{$\phi^{\mathrm{nr}}(1 / 2)$} & $6.6011443629 \times 10^{1}$ & $7.1866675229 \times 10^{1}$ & $7.7466332091 \times 10^{1}$ & $8.2892729834 \times 10^{1}$ & $8.8197557986 \times 10^{1}$ \\
\hline & $13 \mathrm{~d}$ & $14 d$ & $15 \mathrm{~d}$ & $16 \mathrm{~d}$ & $17 \mathrm{~d}$ \\
\hline$a_{0}$ & $-2.6702542838 \times 10^{1}$ & $-2.7142749615 \times 10^{1}$ & $-2.7520108750 \times 10^{1}$ & $-2.7850443691 \times 10^{1}$ & $-2.8146062687 \times 10^{1}$ \\
\hline$\beta$ & $-4.1655350067 \times 10^{-1}$ & $-4.5817894101 \times 10^{-1}$ & $-4.9230994423 \times 10^{-1}$ & $-4.8799319840 \times 10^{-1}$ & $-5.1046103153 \times 10^{-1}$ \\
\hline$b_{0}$ & $1.4031825050 \times 10^{1}$ & $9.3536034158 \times 10^{0}$ & $6.5800387184 \times 10^{0}$ & $6.5003185806 \times 10^{0}$ & $5.0269776925 \times 10^{0}$ \\
\hline$b_{1}$ & $-1.9675880116 \times 10^{1}$ & $-1.9851518345 \times 10^{1}$ & $-2.0183574589 \times 10^{1}$ & $-2.0542531107 \times 10^{1}$ & $-2.0953818322 \times 10^{1}$ \\
\hline$b_{2}$ & $8.0418685007 \times 10^{0}$ & $8.3266612119 \times 10^{0}$ & $8.8233502592 \times 10^{0}$ & $8.8786234533 \times 10^{0}$ & $9.3483218939 \times 10^{0}$ \\
\hline$b_{3}$ & $1.4344987353 \times 10^{1}$ & $1.4507266322 \times 10^{1}$ & $1.4666068986 \times 10^{1}$ & $1.5088704084 \times 10^{1}$ & $1.5370738183 \times 10^{1}$ \\
\hline$b_{4}$ & $6.5426767345 \times 10^{0}$ & $6.3117201027 \times 10^{0}$ & $6.0075843919 \times 10^{0}$ & $6.3138200610 \times 10^{0}$ & $6.1350437791 \times 10^{0}$ \\
\hline$b_{5}$ & $-1.0108197620 \times 10^{1}$ & $-1.0587100158 \times 10^{1}$ & $-1.0920565892 \times 10^{1}$ & $-1.1277066540 \times 10^{1}$ & $-1.1714497606 \times 10^{1}$ \\
\hline$b_{6}$ & $-1.4725216928 \times 10^{0}$ & $-9.1174802515 \times 10^{-1}$ & $-6.0110879243 \times 10^{-1}$ & $-8.1971758897 \times 10^{-1}$ & $-6.0125221865 \times 10^{-1}$ \\
\hline$b_{7}$ & $2.0501069105 \times 10^{0}$ & $2.1660824963 \times 10^{0}$ & $2.4592074550 \times 10^{0}$ & $2.6330961336 \times 10^{0}$ & $2.9489602434 \times 10^{0}$ \\
\hline$b_{8}$ & $-2.0980025116 \times 10^{-1}$ & $-1.8682379725 \times 10^{-1}$ & $-1.5933095829 \times 10^{-1}$ & $-2.0505355745 \times 10^{-1}$ & $-1.7710483631 \times 10^{-1}$ \\
\hline & $2.9129276734 \times 10^{-2}$ & $2.5589737997 \times 10^{-2}$ & $2.1812553698 \times 10^{-2}$ & $3.3518563369 \times 10^{-2}$ & $2.9819941280 \times 10^{-2}$ \\
\hline \multirow[t]{2}{*}{$\phi^{\mathrm{nr}}(1 / 2)$} & $9.3414593519 \times 10^{1}$ & $9.8566665797 \times 10^{1}$ & $1.0366966228 \times 10^{2}$ & $1.0873478648 \times 10^{2}$ & $1.1377060842 \times 10^{2}$ \\
\hline & $18 \mathrm{~d}$ & $19 \mathrm{~d}$ & $20 \mathrm{~d}$ & & \\
\hline$a_{0}$ & $-2.8416405767 \times 10^{1}$ & $-2.8668633507 \times 10^{1}$ & $-2.8908128993 \times 10^{1}$ & & \\
\hline$\beta$ & $-5.2741247590 \times 10^{-1}$ & $-4.8873340484 \times 10^{-1}$ & $-4.7794020321 \times 10^{-1}$ & & \\
\hline$b_{0}$ & $4.1457341691 \times 10^{0}$ & $5.2856466775 \times 10^{0}$ & $5.2735742805 \times 10^{0}$ & & \\
\hline$b_{1}$ & $-2.1898431473 \times 10^{1}$ & $-2.2963670035 \times 10^{1}$ & $-2.3833638246 \times 10^{1}$ & & \\
\hline$b_{2}$ & $1.0391335216 \times 10^{1}$ & $1.1284085887 \times 10^{1}$ & $1.2121030366 \times 10^{1}$ & & \\
\hline$b_{3}$ & $1.6037472635 \times 10^{1}$ & $1.6719978965 \times 10^{1}$ & $1.7273259137 \times 10^{1}$ & & \\
\hline$b_{4}$ & $5.7762172996 \times 10^{0}$ & $5.7383329948 \times 10^{0}$ & $5.5675791948 \times 10^{0}$ & & \\
\hline$b_{5}$ & $-1.2705538272 \times 10^{1}$ & $-1.3194456779 \times 10^{1}$ & $-1.3752468820 \times 10^{1}$ & & \\
\hline$b_{6}$ & $-1.8363323426 \times 10^{-2}$ & $7.2380465060 \times 10^{-2}$ & $3.0584919862 \times 10^{-1}$ & & \\
\hline$b_{7}$ & $3.1624501247 \times 10^{0}$ & $2.7857591072 \times 10^{0}$ & $2.7454079863 \times 10^{0}$ & & \\
\hline$b_{8}$ & $-1.4050292467 \times 10^{-1}$ & $-2.4398422587 \times 10^{-1}$ & $-2.7060311659 \times 10^{-1}$ & & \\
\hline & $2.5244597061 \times 10^{-2}$ & $4.6094366553 \times 10^{-2}$ & $5.2504979483 \times 10^{-2}$ & & \\
\hline$\phi^{\mathrm{nr}}(1 / 2)$ & $1.1878311140 \times 10^{2}$ & $1.2377675855 \times 10^{2}$ & $1.2875544820 \times 10^{2}$ & & \\
\hline
\end{tabular}

